\title{
Total and Partial Computation in Categorical Quantum Foundations
}

\author{
Kenta Cho \\ Institute for Computing and Information Sciences \\ Radboud University, Nijmegen, The Netherlands \\ K.Cho@cs.ru.nl, http://www.cs.ru.nl/K.Cho/
}

\begin{abstract}
This paper uncovers the fundamental relationship between total and partial computation in the form of an equivalence of certain categories. This equivalence involves on the one hand effectuses, which are categories for total computation, introduced by Jacobs for the study of quantum/effect logic. On the other hand, it involves what we call FinPACs with effects; they are finitely partially additive categories equipped with effect algebra structures, serving as categories for partial computation. It turns out that the Kleisli category of the lift monad $(-)+1$ on an effectus is always a FinPAC with effects, and this construction gives rise to the equivalence. Additionally, state-and-effect triangles over FinPACs with effects are presented.
\end{abstract}

\section{Introduction}

An effectus is a category with a final object 1 and finite coproducts $(+, 0)$ satisfying certain assumptions (see Definition 2.2), introduced recently by Jacobs [5], which provides a suitable setting for quantum/effect logic and computation. In an effectus, arrows $\omega: 1 \rightarrow X$ are states on $X$, and $p: X \rightarrow 1+1$ are predicates. They turn out to form a convex set and a so-called effect module, respectively. Arrows $f: X \rightarrow Y$ are seen as computation, inducing state and predicate transformers. The situation is summarised in a state-and-effect triangle, see $\$ 2.2$ for an overview of effectuses.

Motivating examples of effectuses, which model quantum computation and logic, are given by $C^{*}$ algebras with (completely) positive unital maps, and by $W^{*}$-algebras with normal (completely) positive unital maps. Other effectuses include the category Set of sets for a classical setting, and the Kleisli category $\mathcal{K} \ell(\mathcal{D})$ of the distribution monad $\mathcal{D}$ for a probabilistic setting. As seen in these examples, computation modelled by an effectus is total (or terminating) but not partial (or non-terminating). Indeed, arrows in an effectus always induce 'terminating' predicate transformers in the sense that they preserve the truth predicates. We need models of partial computation in some cases, however, since programs do not necessarily terminate in general. Moreover, such models often have richer structures such as complete partial orders, which allow us to interpret loop and recursion. For instance, the category of sets and partial functions are enriched over complete partial orders, and so is the category of $W^{*}$-algebras and normal (completely) positive subunital maps [2,11].

The present paper studies partial computation in effectuses via the lift monad (a.k.a. maybe monad), which is a common technique in categorical semantics of computation, going back to Moggi [9]. We switch from an effectus $\mathbf{B}$ to the Kleisli category of the lift monad $(-)+1$ on $\mathbf{B}$, which we denote by $\mathbf{B}_{+1}$. An arrow $X \rightarrow Y$ in $\mathbf{B}_{+1}$ is $X \rightarrow Y+1$ in $\mathbf{B}$, seen as a partial computation from $X$ to $Y$. This simple idea makes a lot of sense for any effectus, leading us to the main results of this paper as follows.

- For an effectus $\mathbf{B}$, the Kleisli category $\mathbf{B}_{+1}$ of the lift monad is a finitely partially additive category (FinPAC), which is a finite variant of Arbib and Manes' partially additive category (PAC) [1, 8].

Chris Heunen, Peter Selinger, and Jamie Vicary (Eds.):

12th International Workshop on Quantum Physics and Logic (QPL 2015).

EPTCS 195, 2015, pp. 116-135 doi 10.4204/EPTCS.195.9

(C) K. Cho 
Table 1: Examples of effectuses and corresponding FinPACs with effects.

\begin{tabular}{cccc} 
effectus & total computation & partial computation & FinPAC with effects \\
\hline \hline $\mathbf{B}$ & $X \rightarrow Y$ & $X \rightarrow Y+1$ (in $\mathbf{B})$ & $\mathbf{B}_{+1}$ \\
\hline Set & function & partial function & Pfn \\
\hline $\mathcal{K} \ell(\mathcal{D})$ & $\begin{array}{c}X \rightarrow \mathcal{D} Y \\
\text { (stochastic relation) }\end{array}$ & $\begin{array}{c}X \rightarrow \widehat{\mathcal{D}} Y \\
\text { (substochastic relation) }\end{array}$ & $\mathcal{K} \ell(\widehat{\mathcal{D}})$ \\
\hline Cstar $\left._{\mathrm{PU}}\right)^{\text {op }}$ & positive unital map & positive subunital map & $\left(\mathbf{C s t a r}_{\mathrm{PSU}}\right)^{\text {op }}$
\end{tabular}

The homsets $\mathbf{B}_{+1}(X, 1)=\mathbf{B}(X, 1+1)$ are the sets of predicates and form effect algebras. The category $\mathbf{B}_{+1}$ is what we call a FinPAC with effects, which has an effect algebra structure related to the partially additive structure in an appropriate manner (see Definition 4.4).

- On the other hand, if $\mathbf{C}$ is a FinPAC with effects, then the subcategory $\mathbf{C}_{t}$ with 'total' arrows is an effectus. Moreover, the two constructions $(-)_{+1}$ and $(-)_{t}$ are inverses of each other up to isomorphism. Categorically, we obtain a 2-equivalence of the 2-categories of effectuses and FinPACs with effects.

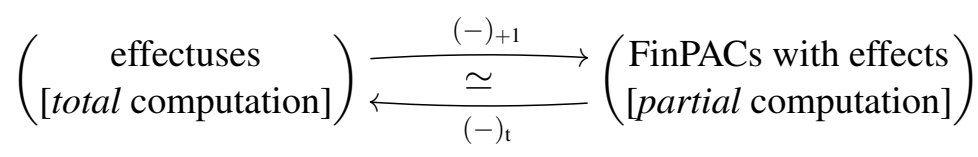

See Table 1 for examples of effectuses and corresponding FinPACs with effects. This equivalence characterises the Kleisli categories $\mathbf{B}_{+1}$ of the lift monad on effectuses as FinPACs with effects, and effectuses as the 'total' subcategories $\mathbf{C}_{t}$ of FinPACs with effects.

We additionally present two type of state-and-effect triangles over a FinPAC with effects. One triangle is rather simple and easy, involving generalised effect modules and subconvex sets. Another triangle is obtained by an application of the above 2-equivalence to a state-and-effect triangle over an effectus, but only under an additional 'normalisation' condition. This also contains a slight improvement of a known result on effectuses with normalisation, via division in effect monoids.

The paper is organised as follows. We first give preliminaries in the next section. Section 3 introduces FinPACs. In $\$ 4$ we study partial computation in effectuses and FinPACs with effects, and then in $\$ 5$ we prove categorical equivalence of effectuses and FinPACs with effects. Section 6 presents state-and-effect triangles over FinPACs with effects.

\section{Preliminaries}

\subsection{Partial commutative monoids, (generalised) effect modules and (sub)convex sets}

A partial commutative monoid ( $P C M)$ is a set $M$ with a partial binary 'sum' operation $\oslash: M \times M \rightarrow M$ and a 'zero' element $0 \in M$ subject to $(x \otimes y) \oslash z \simeq x \otimes(y \otimes z), x \otimes y \simeq y \otimes x$ and $x \otimes 0 \simeq x$, where $\simeq$ denotes the Kleene equality: if either side is defined, then so is the other, and they are equal. We write $x \perp y$ if $x \otimes y$ is defined, and we say elements $x_{1}, \ldots, x_{n}$ are orthogonal if $x_{1} \oslash x_{2} \oslash \cdots \otimes x_{n}$ is defined. Any PCM carries a preorder via $x \leq y \Leftrightarrow \exists z \cdot x \otimes z=y$, with 0 as a bottom (a least element). A generalised effect algebra (GEA) is a PCM that is positive $(x \otimes y=0 \Rightarrow x=y=0)$ and cancellative $(x \otimes y=x \otimes z \Rightarrow y=z)$. In a GEA the preorder $\leq$ above is a partial order, and we have a 'partial difference' given by $x \ominus y=z \Leftrightarrow x=y \oslash z$. An effect algebra is a GEA that has a top (a greatest element), 
which is denoted by 1 . Any element $x$ in an effect algebra has an orthocomplement $x^{\perp}:=1 \ominus x$, i.e. a unique element such that $x \otimes x^{\perp}=1$. Homomorphisms of PCMs and GEAs preserve $\oslash$ and 0 , and those of effect algebras additionally preserve 1 . An effect monoid is an effect algebra $M$ with a 'multiplication' PCM-bihomomorphism $:: M \times M \rightarrow M$ satisfying $1 \cdot r=r=r \cdot 1$ and $(r \cdot s) \cdot t=r \cdot(s \cdot t)$. A partial commutative module (PCMod) over an effect monoid $M$ is a PCM $E$ with a 'scalar multiplication' PCMbihomomorphism $\bullet: M \times E \rightarrow E$ satisfying $1 \bullet x=x$ and $r \bullet(s \bullet x)=(r \cdot s) \bullet x$. A generalised effect module (GEMod) and an effect module are respectively a GEA and an effect algebra that are at the same time a PCMod. Homomorphisms of them are required to preserve the scalar multiplication.

For an effect monoid $M$, we denote by $\mathcal{D}_{M}$ and $\widehat{\mathcal{D}}_{M}$ respectively the (finite, discrete) distribution and subdistribution monads over $M$ on the category Set. For a set $X$, the set $\mathcal{D}_{M} X$ consists of formal convex sums $\left|x_{1}\right\rangle r_{1}+\cdots+\left|x_{n}\right\rangle r_{n}$ where $x_{i} \in X$ and $r_{i} \in M$ with $\emptyset_{i} r_{i}=1$, while $\widehat{\mathcal{D}}_{M} X$ consists of $\left|x_{1}\right\rangle r_{1}+$ $\cdots+\left|x_{n}\right\rangle r_{n}$ with $\emptyset_{i} r_{i} \leq 1$ (which holds automatically as long as $r_{1}, \ldots, r_{n}$ are orthogonal). Here the 'ket' notation $|x\rangle$ is just syntactic sugar to distinguish formal sums from elements $x \in X$. A convex set (resp. subconvex set) over $M$ is an Eilenberg-Moore algebra of $\mathcal{D}_{M}$ (resp. $\widehat{\mathcal{D}}_{M}$ ), which is a set $X$ with an operation mapping a formal sum $\sum_{i}\left|x_{i}\right\rangle r_{i} \in \mathcal{D}_{M} X$ (resp. $\left.\widehat{\mathcal{D}}_{M} X\right)$ to an actual sum $\emptyset_{i} x_{i} r_{i} \in X$. For an effect $\operatorname{monoid}_{M}$ we write $\operatorname{EMod}_{M}$ and $\operatorname{GEMod}_{M}$ for the categories of effect modules and GEMod's over $M$, and $\operatorname{Conv}_{M}=\mathcal{E M}\left(\mathcal{D}_{M}\right)$ and $\operatorname{SConv}_{M}=\mathcal{E M}\left(\widehat{\mathcal{D}}_{M}\right)$ for the categories of convex and subconvex sets over $M$. The following dualities are fundamental.

Proposition 2.1. There are the following adjunctions, obtained by "homming into M".

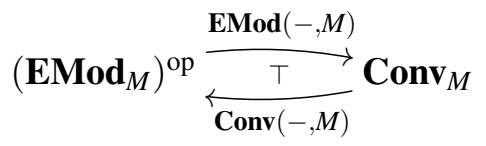

$\left(\operatorname{GEMod}_{M}\right)^{\text {op }} \underset{\operatorname{SConv}(-, M)}{\stackrel{\operatorname{GEMod}(-, M)}{\longrightarrow}} \operatorname{SConv}_{M}$

Proof. The left-hand adjunction is shown in [5, Proposition 6]. The right-hand one is shown in [10, Appendix B] for $M=[0,1]$, and the proof is easily generalised.

We say a PCMod $E$ over an effect monoid $M$ is subconvex if $r_{1} \bullet x_{1}, \cdots, r_{n} \bullet x_{n}$ are orthogonal for any $x_{1}, \cdots, x_{n} \in E$ and for orthogonal $r_{1}, \cdots, r_{n} \in M$. A subconvex PCMod is then a subconvex set via the subconvex sum $\emptyset_{i} r_{i} \bullet x_{i}$. The category PCM of PCMs is symmetric monoidal closed via a tensor product representing bihomomorphisms [6]. Therefore, PCM-enriched categories are well-defined. Explicitly, a category is PCM-enriched if each homset is a PCM and the composition is a PCM-bihomomorphism.

\subsection{Effectuses}

Several assumptions on a category were identified by Jacobs [5] for the study of quantum/effect logic and computation. A category that satisfies the most basic assumption [5. Assumption 1] is now called an 'effectus', since [7]:1]

Definition 2.2. An effectus is a category with a final object 1 and finite coproducts $(+, 0)$ satisfying:

- squares of the following form (E) and $\left(\mathrm{K}^{=}\right)$are pullbacks;

$$
\begin{gathered}
A+X \stackrel{\mathrm{id}+f}{\longrightarrow} A+Y \\
g+\mathrm{id} \downarrow \\
B+X \underset{\mathrm{id}+f}{\downarrow} B+Y
\end{gathered}
$$

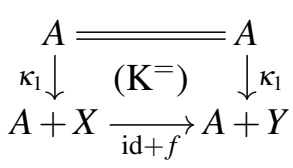

\footnotetext{
${ }^{1}$ Note that [7. Definition 12] uses a slightly different joint monicity requirement. In the present paper we follow [5].
} 
- the two arrows $\left[\kappa_{1}, \kappa_{2}, \kappa_{2}\right],\left[\kappa_{2}, \kappa_{1}, \kappa_{2}\right]: 1+1+1 \rightarrow 1+1$ are jointly monic.

In an effectus $\mathbf{B}$, a state on an object $X$ is an arrow $\omega: 1 \rightarrow X$; a predicate on $X$ is $p: X \rightarrow 1+1$; and a scalar is $r: 1 \rightarrow 1+1$. For a state $\omega$ and a predicate $p$, the validity probability is given by the abstract Born rule $(\omega \vDash p):=p \circ \omega: 1 \rightarrow 1+1$. We write $\operatorname{Stat}(X)=\mathbf{B}(1, X)$ and $\operatorname{Pred}(X)=\mathbf{B}(X, 1+1)$ for the sets of states and predicates respectively.

It turns out that the set of scalars $M:=\mathbf{B}(1,1+1)$ is an effect monoid, and $\operatorname{Stat}(X)$ and $\operatorname{Pred}(X)$ are a convex set and an effect module over $M$ respectively. In particular, $\operatorname{Pred}(X)$ is a poset with a top (truth) $1_{X}$ and a bottom (falsum) $0_{X}$. We refer to [5] for the details, but later in $\$ 6$ we will come to this point from a 'partial' perspective. An arrow $f: X \rightarrow Y$ in $\mathbf{B}$ induces a state transformer $\operatorname{Stat}(f): \operatorname{Stat}(X) \rightarrow \operatorname{Stat}(Y)$ by $\operatorname{Stat}(f)(\omega)=f \circ \omega$,

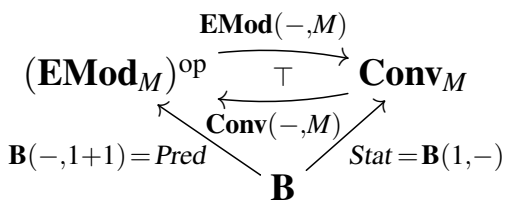

Figure 1: State-and-effect triangle and a (backward) predicate transformer Pred $(f): \operatorname{Pred}(Y) \rightarrow \operatorname{Pred}(X)$ by $\operatorname{Pred}(f)(p)=p \circ f$, making Stat and Pred functors in a state-and-effect triangle shown in Figure 1 .

The dual adjunction $\left(\mathbf{E M o d}_{M}\right)^{\text {op }} \rightleftarrows \mathbf{C o n v}_{M}$ from Proposition 2.1 expresses the duality between predicates and states. By "currying" the abstract Born rule $\vDash: \operatorname{Stat}(X) \times \operatorname{Pred}(X) \rightarrow M$ we obtain maps $\alpha_{X}$ and $\beta_{X}$ in the bijective correspondence of the dual adjunction:

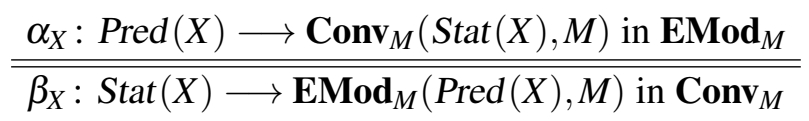

These maps $\alpha$ and $\beta$ are natural transformations filling the triangle.

A motivating example of an effectus, which models quantum computation and logic, is the opposite $\left(\text { Cstar }_{\mathrm{PU}}\right)^{\text {op }}$ of the category of (unital) $C^{*}$-algebras and positive unital (PU) maps. Note that an initial

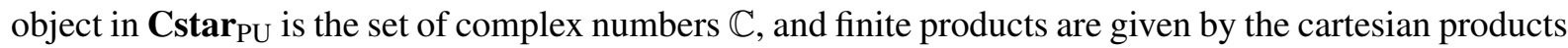
of underlying sets with coordinatewise operations; they are a final object and finite coproducts in the opposite. Then, states on a $C^{*}$-algebra $A$ are PU-maps $\omega: A \rightarrow \mathbb{C}$, which coincide with the standard definition of 'states' in operator theory. Predicates on $A$ are PU-maps $f: \mathbb{C} \times \mathbb{C} \rightarrow A$, which are in bijective correspondence with elements $p \in A$ with $0 \leq p \leq 1$, via $p=f(1,0)$ and $f(\lambda, \rho)=\lambda p+\rho(1-$ $p$ ). Such elements $p \in A$ with $0 \leq p \leq 1$ are called effects and thought of as "unsharp" predicates, which include "sharp" projections. Scalars are effects in the complex numbers $\mathbb{C}$, i.e. real numbers between 0 and 1. Then the abstract Born rule is the usual Born rule $(\omega \vDash p):=\omega(p) \in[0,1]$. The sum $\emptyset$ of effects $p, q$ is defined if $p+q \leq 1$; and in that case $p \otimes q=p+q$. With an obvious scalar multiplication, effects form an effect module. The convex structure of states $\omega: A \rightarrow \mathbb{C}$ is given in a pointwise manner. One has similar examples of effectuses given by $C^{*}$-algebras with completely positive unital maps, and $W^{*}$-algebras with normal (completely) positive unital maps.

Another example of an effectus is the category Set of sets and functions, which models classical computation and logic. States $1 \rightarrow X$ are simply elements $x \in X$, while predicates $X \rightarrow 1+1 \cong 2$ are subsets $P \subseteq X$ as usual. The set of scalars is the two element set $\{0,1\}$, and then the abstract Born rule is the membership relation $(x \vDash P)=(x \in P)$. The Kleisli category $\mathcal{K} \ell(\mathcal{D})$ of the distribution monad $\mathcal{D}=\mathcal{D}_{[0,1]}$ over $[0,1]$ is an effectus for a probabilistic setting. States $1 \rightarrow X$ are functions $1 \rightarrow \mathcal{D}(X)$, hence probability distributions $\omega \in \mathcal{D}(X)$. Predicates $X \rightarrow 1+1$ are functions $X \rightarrow \mathcal{D}(1+1) \cong[0,1]$, thus 'fuzzy' predicates $p \in[0,1]^{X}$. The set of scalars is the unit interval $[0,1]$, and the abstract Born rule is given by the expectation value $(\omega \vDash p)=\sum_{x} p(x) \omega(x)$.

Further explanation and examples are found in [5]. 


\section{Finitely partially additive categories (FinPACs)}

Here we introduce a notion of finitely partially additive category (FinPAC), which is a finite variant of Arbib and Manes' partially additive category (PAC) [1, 8]. The difference is that a PAC involves countable sums, but a FinPAC involves only finite sums.

We first need a few preliminary definitions. A category has zero arrows if there is a family of 'zero arrows' $0_{X Y}: X \rightarrow Y$ such that $0_{W Y} \circ f=0_{X W}=g \circ 0_{X Z}$ for all $f: X \rightarrow Y$ and $g: Z \rightarrow W$. Such a family is unique if exists (indeed, $0_{X Y}=0_{X Y} \circ 0_{X X}^{\prime}=0_{X Y}^{\prime}$ ). If a category has a zero object 0 , then it has zero arrows $X \rightarrow 0 \rightarrow Y$. The converse is also true when the category has an initial (or final) object, see e.g. [8, §2.2.19]. For a coproduct $\coprod_{i \in I} X_{i}$ in a category with zero arrows, we define partial projections ${ }^{2}$ $\triangleright_{i}: \coprod_{i \in I} X_{i} \rightarrow X_{i}$ by $\triangleright_{i} \circ \kappa_{i}=\mathrm{id}_{X_{i}}$ and $\triangleright_{i} \circ \kappa_{j}=0_{X_{j} X_{i}}(j \neq i)$.

Definition 3.1 (cf. [1, §3.3]). A finitely partially additive category (FinPAC for short) is a category $\mathbf{C}$ with finite coproducts $(+, 0)$ which is PCM-enriched and satisfies the following two axioms.

- (Compatible sum axiom) Arrows $f, g: X \rightarrow Y$ are orthogonal (in the PCM $\mathbf{C}(X, Y)$ ) whenever $f$ and $g$ are compatible in the sense that there exists a 'bound' $b: X \rightarrow Y+Y$ such that $f=\triangleright_{1} \circ b$ and $g=\triangleright_{2} \circ b$.

- (Untying axiom) If $f, g: X \rightarrow Y$ are orthogonal, then $\kappa_{1} \circ f, \kappa_{2} \circ g: X \rightarrow Y+Y$ are orthogonal too. Note that $\mathbf{C}$ has zero arrows, i.e. zero elements $0_{X Y}$ of the PCMs $\mathbf{C}(X, Y)$.

For any objects $Y_{1}$ and $Y_{2}$ in a FinPAC, arrows $\kappa_{1} \circ \triangleright_{1}, \kappa_{2} \circ \triangleright_{2}: Y_{1}+Y_{2} \rightarrow Y_{1}+Y_{2}$ are compatible via a bound $\kappa_{1}+\kappa_{2}$, hence orthogonal. Then we obtain $\kappa_{1} \circ \triangleright_{1} \otimes \kappa_{2} \circ \triangleright_{2}=\operatorname{id}_{Y_{1}+Y_{2}}$ because $\left(\kappa_{1} \circ \triangleright_{1} \oslash\right.$ $\left.\kappa_{2} \circ \triangleright_{2}\right) \circ \kappa_{i}=\kappa_{i}$. For any arrow $f: X \rightarrow Y_{1}+Y_{2}$, therefore, one has a 'decomposition' $f=\left(\kappa_{1} \circ \triangleright_{1} \otimes\right.$ $\left.\kappa_{2} \circ \triangleright_{2}\right) \circ f=\kappa_{1} \circ f_{1} \oslash \kappa_{2} \circ f_{2}$, where $f_{i}=\triangleright_{i} \circ f: X \rightarrow Y_{i}$. It then easily follows that the two partial projections $\triangleright_{i}: Y_{1}+Y_{2} \rightarrow Y_{i}$ are jointly monic: $\triangleright_{i} \circ f=\triangleright_{i} \circ g$ for $i=1,2$ implies $f=g$. Now we see the PCM-enrichment of a FinPAC is unique, as is the case for a PAC (cf. [8, Theorem 3.2.18]).

Proposition 3.2. In a FinPAC, arrows $f, g: X \rightarrow Y$ are orthogonal if and only if they are compatible. In that case, a bound $b: X \rightarrow Y+Y$ for $f$ and $g$ is unique and $f \otimes g=\nabla \circ b$, where $\nabla=[\mathrm{id}$,id] is the codiagonal.

Proof. Assume that $f$ and $g$ are orthogonal. By untying, $\kappa_{1} \circ f, \kappa_{2} \circ g: X \rightarrow Y+Y$ are orthogonal, and then let $b=\kappa_{1} \circ f \otimes \kappa_{2} \circ g$. It is easy to see that $f$ and $g$ are compatible via $b$. A bound $b: X \rightarrow Y+Y$ is unique since $\triangleright_{1}$ and $\triangleright_{2}$ are jointly monic. Finally, $\nabla \circ b=\nabla \circ\left(\kappa_{1} \circ f \otimes \kappa_{2} \circ g\right)=f \otimes g$.

In a FinPAC, not very surprisingly, the $n$-ary version of the compatible sum and the untying axiom hold, proved by induction with a small trick; see Lemma A.1. Therefore, we have the decomposition property for finite coproducts, shown in the same way as the binary case above.

Lemma 3.3. Let $\coprod_{i} Y_{i}$ be a finite coproduct in a FinPAC. Any arrow $f: X \rightarrow \coprod_{i} Y_{i}$ is uniquely decomposed as $f=\emptyset_{i} \kappa_{i} \circ f_{i}$, where $f_{i}=\triangleright_{i} \circ f: X \rightarrow Y_{i}$. Thus, the partial projections $\triangleright_{i}: \coprod_{i} Y_{i} \rightarrow Y_{i}$ are jointly monic.

Finally, we give a characterisation of FinPACs.

Theorem 3.4 (cf. [1, §5.3]). A category is a FinPAC if and only if it has finite coproducts and zero arrows, and satisfies the following two conditions for each object $X$ :

- the two partial projections $\triangleright_{1}, \triangleright_{2}: X+X \rightarrow X$ are jointly monic;

- the square on the right is a pullback.

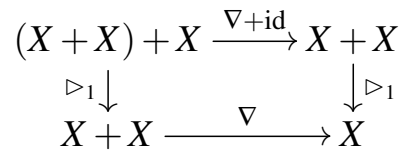

${ }^{2}$ Arbib and Manes call them quasi projections. 
Proof. For the 'if' direction, we define the partial sum $\oslash$ in the manner of Proposition 3.2. The complete proof is deferred to Appendix A.

\section{Partial computation in effectuses and FinPACs with effects}

Recall from Definition 2.2 that an effectus $\mathbf{B}$ has a final object 1 and finite coproducts $(+, 0)$. Therefore we have the lift monad $(-)+1$ on $\mathbf{B}$. The unit is the first coprojection $\kappa_{1}: X \rightarrow X+1$; the multiplication is the cotuple $\left[\operatorname{id}_{X+1}, \kappa_{2}\right]:(X+1)+1 \rightarrow X+1$; and the Kleisli extension of $f: X \rightarrow Y+1$ is the cotuple $\left[f, \kappa_{2}\right]: X+1 \rightarrow Y+1$. Its Kleisli category, seen as a category for partial computation, plays an important role in this paper. Hence we reserve a few notations for it.

We denote by $\mathbf{B}_{+1}$ the Kleisli category of the lift monad on $\mathbf{B}$. Namely, $\mathbf{B}_{+1}$ has the same objects as $\mathbf{B}$, and arrows given by $\mathbf{B}_{+1}(X, Y)=\mathbf{B}(X, Y+1)$. We write $f: X \rightarrow Y$ ('harpoon' arrows) for arrows in $\mathbf{B}_{+1}$, and $g \hat{\circ} f=\left[g, \kappa_{2}\right] \circ f$ for the composition in $\mathbf{B}_{+1}$. We denote the canonical functor $\mathbf{B} \rightarrow \mathbf{B}_{+1}$ by $(\widehat{-})$; namely $\widehat{X}=X$ and $\widehat{f}=\kappa_{1} \circ f$. Then $\widehat{\mathrm{id}}_{X}$ denotes the identity $\kappa_{1}: X \rightarrow X$ in $\mathbf{B}_{+1}$. The Kleisli category $\mathbf{B}_{+1}$ has all finite coproducts, which are inherited from $\mathbf{B}$ in the way the functor $(\widehat{-}): \mathbf{B} \rightarrow \mathbf{B}_{+1}$ preserves the coproducts on the nose. In other words, a coproduct in $\mathbf{B}_{+1}$ is a coproduct $\coprod_{i} X_{i}$ in $\mathbf{B}$ with coprojections $\widehat{\kappa}_{i}: X_{i} \rightarrow \coprod_{i} X_{i}$, where $\kappa_{i}$ are coprojections in $\mathbf{B}$. For arrows $f, g$ in $\mathbf{B}_{+1}$, we write $f \hat{+} g=\left[\widehat{\kappa}_{1} \hat{\circ} f, \widehat{\kappa}_{2} \hat{\circ} g\right]$ in order to distinguish it from $f+g$ in $\mathbf{B}$. The base category $\mathbf{B}$ is understood as the 'total' part of $\mathbf{B}_{+1}$ via $(\widehat{-}): \mathbf{B} \rightarrow \mathbf{B}_{+1}$, see also Proposition 4.6 .

We first collect basic facts on an effectus $\mathbf{B}$ and the Kleisli category $\mathbf{B}_{+1}$.

Lemma 4.1. Let $\mathbf{B}$ be an effectus.

1. In $\mathbf{B}$, coprojections $\kappa_{i}$ are monic. Thus, the functor $(\widehat{-}): \mathbf{B} \rightarrow \mathbf{B}_{+1}$ is faithful.

2. In $\mathbf{B}$, squares of the form $(K)$ below are pullbacks, generalising $\left(K^{=}\right)$.

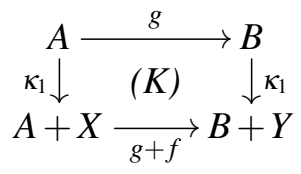

3. $\mathbf{B}_{+1}$ has zero arrows $0_{X Y}: X \rightarrow Y$ given by $X \stackrel{!_{X}}{\longrightarrow} 1 \stackrel{\kappa_{2}}{\longrightarrow} Y+1$ in $\mathbf{B}$.

4. In $\mathbf{B}_{+1}$, the partial projections $\triangleright_{1}, \triangleright_{2}: X+X \rightarrow X$ are jointly monic.

5. For a ('total') arrow $f: X \rightarrow Y$ in $\mathbf{B}$, the following square is a pullback in $\mathbf{B}_{+1}$.

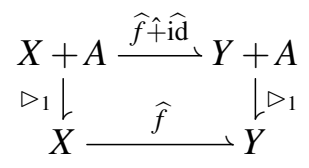

Proof. For 1 and 2, see [5, Lemma 10].

3. Straightforward.

4. It holds precisely when $\left[\triangleright_{1}, \kappa_{2}\right],\left[\triangleright_{2}, \kappa_{2}\right]:(X+X)+1 \rightarrow X+1$ are jointly monic in $\mathbf{B}$, which is indeed the case; see [5, Lemma 11]. 
5. The square is a pullback in $\mathbf{B}_{+1}$ if and only if the left-hand square below is pullback in $\mathbf{B}$.

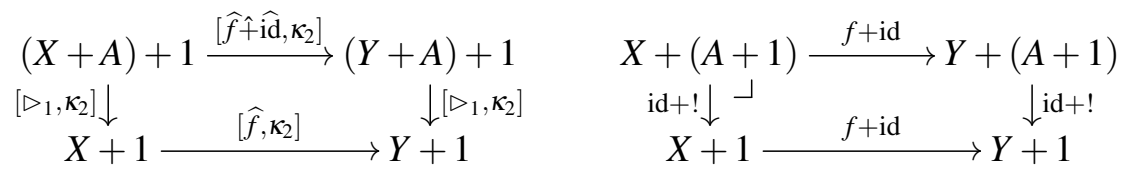

Up to isomorphism, it coincides with the right-hand pullback (E).

Theorem 4.2. For an effectus $\mathbf{B}$, the category $\mathbf{B}_{+1}$ is a FinPAC.

Proof. We use Theorem 3.4, a characterisation of a FinPAC. We have already seen that $\mathbf{B}_{+1}$ has finite coproducts and zero arrows, and that the partial projections $\triangleright_{1}, \triangleright_{2}: X+X \rightarrow X$ are jointly monic. The required pullback is an instance of Lemma 4.15 via $f=\nabla: X+X \rightarrow X$.

Thus, $\mathbf{B}_{+1}$ is $\mathbf{P C M}$-enriched, and in particular the sets of predicates $\mathbf{B}(X, 1+1)=\mathbf{B}_{+1}(X, 1)$ are PCMs. Jacobs showed that predicates have even more structures.

Proposition 4.3 ([5, Proposition 13]). Let $\mathbf{B}$ be an effectus. For each $X \in \mathbf{B}$, the homset $\mathbf{B}(X, 1+1)=$ $\mathbf{B}_{+1}(X, 1)$ is an effect algebra with the top $1_{X}:=\kappa_{1} \circ !_{X}=\widehat{!}_{X}$.

Note that the PCM structure on predicates given by Jacobs [5, Definition 12] coincides with our partially additive structure (see Proposition 3.2). Crucially, the category $\mathbf{B}_{+1}$ is not only equipped with both the partially additive and the effect algebra structure, but satisfies suitable conditions that relate them. We give a name to such categories, since they will turn out to characterise $\mathbf{B}_{+1}$.

Definition 4.4. A FinPAC with effects is a FinPAC $\mathbf{C}$ with a special object $I \in \mathbf{C}$ such that hom-PCMs $\mathbf{C}(X, I)$ are effect algebras for all $X \in \mathbf{C}$, satisfying the two conditions below. We write $1_{X}$ and $0_{X}\left(=0_{X I}\right)$ for the top and the bottom of $\mathbf{C}(X, I)$.

1. $1_{Y} \circ f=0_{X}$ implies $f=0_{X Y}$ for all $f: X \rightarrow Y$.

2. $1_{Y} \circ f \perp 1_{Y} \circ g$ implies $f \perp g$ for all $f, g: X \rightarrow Y$.

Theorem 4.5. Let $\mathbf{B}$ be an effectus. Then $\left(\mathbf{B}_{+1}, 1\right)$ is a FinPAC with effects.

Proof. We check the two requirement. 1) Assume that $1_{Y} \hat{\circ} f=0_{X}$, i.e. $\left(!_{Y}+\right.$ id $) \circ f=\kappa_{2} \circ !_{X}$. Using a pullback $(\mathrm{K})$ with the symmetry of coproducts, we obtain $f=\kappa_{2} \circ !_{X}=0_{X Y}$ as in the left diagram below.
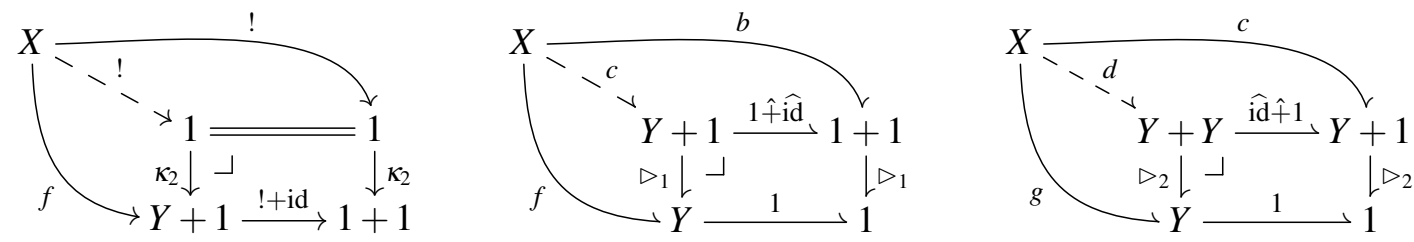

2) Let $b: X \rightarrow 1+1$ be a bound for $1_{Y} \hat{\circ} f$ and $1_{X} \hat{o} g$. Note that $1_{Y}=\hat{!}_{Y}$ is a 'total' arrow. Then we use a pullback of Lemma 4.15 and obtain a mediating map $c: X \rightarrow Y+1$ as in the middle diagram above. Using a similar pullback given by the symmetry of coproducts, we obtain $d: X \rightarrow Y+Y$ as in the right diagram above. Then it is straightforward to check $d$ is a bound for $f$ and $g$.

In a FinPAC with effects $(\mathbf{C}, I)$, we call an arrow $p: X \rightarrow I$ a predicate on $X$, and write Pred $(X)=$ $\mathbf{C}(X, I)$, which is by definition an effect algebra. When $\mathbf{C}=\mathbf{B}_{+1}$, this definition coincides with predicates in the effectus $\mathbf{B}$, since $\mathbf{B}_{+1}(X, 1)=\mathbf{B}(X, 1+1)$. For an arrow $f: X \rightarrow Y$ in $\mathbf{C}$, we call $1_{Y} \circ f \in$ Pred $(X)$ the domain predicate of $f$ and write $\operatorname{Dp}(f)=1_{Y} \circ f$. We then have a PCM-homomorphism Dp: $\mathbf{C}(X, Y) \rightarrow \operatorname{Pred}(X)$. We say an arrow $f: X \rightarrow Y$ in $\mathbf{C}$ is total if $\operatorname{Dp}(f)=1_{X}$. It is easy to see that all objects of $\mathbf{C}$ with total arrows form a subcategory of $\mathbf{C}$, which is denoted by $\mathbf{C}_{\mathrm{t}}$. 
Proposition 4.6. For an effectus $\mathbf{B}$, the functor $(\widehat{-}): \mathbf{B} \rightarrow \mathbf{B}_{+1}$ restricts to an isomorphism $\mathbf{B} \cong\left(\mathbf{B}_{+1}\right)_{\mathrm{t}}$.

Proof. Since $(\widehat{-})$ is faithful, it suffices to show that an arrow $f: X \rightarrow Y$ in $\mathbf{B}_{+1}$ is total if and only if $f=\widehat{g}$ for some $g \in X \rightarrow Y$ in $\mathbf{B}$. If $f=\widehat{g}$ then $\operatorname{Dp}(f)=1_{Y} \hat{\circ} f=\widehat{!}_{Y} \hat{\circ} \widehat{g}=\widehat{!_{Y} \circ g}=1_{X}$. Conversely, assume that $\operatorname{Dp}(f)=1_{X}$, i.e. $\left(!_{Y}+\mathrm{id}\right) \circ f=\kappa_{1} \circ !_{X}$. Using a pullback $(\mathrm{K})$, we obtain $g: X \rightarrow Y$ with $f=\kappa_{1} \circ g=\widehat{g}$.

We list several basic properties of a FinPAC with effects.

Lemma 4.7. In a FinPAC with effects $(\mathbf{C}, I)$, the following hold.

1. $f=0_{X Y}$ if and only if $\operatorname{Dp}(f)=0_{X}$ for all $f: X \rightarrow Y$.

2. $f_{1}, \ldots, f_{n}$ are orthogonal if and only if $\mathrm{Dp}\left(f_{1}\right), \ldots, \mathrm{Dp}\left(f_{n}\right)$ are orthogonal for all $f_{1}, \ldots, f_{n}: X \rightarrow Y$. In that case, $\mathrm{Dp}\left(\bigotimes_{i} f_{i}\right)=\bigotimes_{i} \mathrm{Dp}\left(f_{i}\right)$.

3. $\operatorname{Dp}(g \circ f)=\operatorname{Dp}(g) \circ f \leq \operatorname{Dp}(f)$ for all $f: X \rightarrow Y$ and $g: Y \rightarrow Z$. If $g$ is total then $\operatorname{Dp}(g \circ f)=$ $\operatorname{Dp}(f)$.

4. Any split mono is total. In particular, any isomorphism is total.

5. Coprojections $\kappa_{i}$ are split monic and hence total.

6. $1_{I}=\mathrm{id}_{I}: I \rightarrow I$.

Proof. 1. The 'only if' direction holds because Dp is homomorphism, while 'if' holds by definition.

2. The binary case is immediate, like 1 . Then the $n$-ary case follows by induction.

3. $\operatorname{Dp}(g \circ f)=1_{Z} \circ g \circ f=\operatorname{Dp}(g) \circ f \leq 1_{Y} \circ f=\operatorname{Dp}(f)$. We have equality when $g$ is total.

4. If $g \circ f=\mathrm{id}$, then $\operatorname{Dp}(f) \geq \operatorname{Dp}(g \circ f)=\operatorname{Dp}(\mathrm{id})=1$.

5. Coprojections are split monic as $\triangleright_{i} \circ \kappa_{i}=\mathrm{id}$.

6. Note that $\operatorname{Dp}\left(\operatorname{id}_{I}\right) \perp \operatorname{Dp}\left(\operatorname{id}_{I}^{\perp}\right)$ and $\operatorname{Dp}\left(\operatorname{id}_{I}\right)=1_{I}$. Then $\operatorname{Dp}\left(\operatorname{id}_{I}^{\perp}\right)=0_{I}$ and hence $\operatorname{id}_{I}^{\perp}=0_{I I}=0_{I}$. Namely $\operatorname{id}_{I}=1_{I}$.

In a FinPAC with effects, we have the decomposition property (Lemma 3.3) as a more explicit bijective correspondence involving domain predicates.

Lemma 4.8. Let $\coprod_{i} Y_{i}$ be a finite coproduct in a FinPAC with effects. We have the following bijective correspondence.

$$
\frac{\text { an arrow } f: X \longrightarrow \coprod_{i} Y_{i}}{\text { a family } \left.\left(f_{i}: X \longrightarrow Y_{i}\right)_{i} \text { where }\left(\operatorname{Dp}\left(f_{i}\right)\right)_{i} \text { is orthogonal (so that } \emptyset_{i} \operatorname{Dp}\left(f_{i}\right) \leq 1_{X}\right)}
$$

They are related in $f_{i}=\triangleright_{i} \circ f$ and $f=\bigotimes_{i} \kappa_{i} \circ f_{i}$. Moreover one has $\operatorname{Dp}(f)=\bigotimes_{i} \operatorname{Dp}\left(f_{i}\right)$. In particular, $f$ is total if and only if $\emptyset_{i} \operatorname{Dp}\left(f_{i}\right)=1_{X}$.

Proof. Given $f: X \rightarrow \coprod_{i} Y_{i}$, we have the decomposition $f=\emptyset_{i} \kappa_{i} \circ f_{i}$, where $f_{i}=\triangleright_{i} \circ f$. The family $\left(\operatorname{Dp}\left(f_{i}\right)\right)_{i}$ is orthogonal because $\operatorname{Dp}\left(f_{i}\right)=\operatorname{Dp}\left(\kappa_{i} \circ f_{i}\right)$. Conversely, if $\left(\operatorname{Dp}\left(f_{i}\right)\right)_{i}$ is orthogonal for arrows $f_{i}: X \rightarrow Y_{i}$, then $\left(\kappa_{i} \circ f_{i}\right)_{i}$ is orthogonal. Hence we have the sum $f=\bigotimes_{i} \kappa_{i} \circ f_{i}$. It is easy to see that the correspondence is bijective. Finally, $\operatorname{Dp}(f)=\emptyset_{i} \operatorname{Dp}\left(\kappa_{i} \circ f_{i}\right)=\emptyset_{i} \operatorname{Dp}\left(f_{i}\right)$.

Lemma 4.9. Let $(\mathbf{C}, I)$ be a FinPAC with effects. Coproducts $\coprod_{i} X_{i}$ in $\mathbf{C}$ restrict to $\mathbf{C}_{\mathrm{t}}$, so that $\mathbf{C}_{\mathrm{t}}$ has all finite coproducts. Moreover, I is final in $\mathbf{C}_{\mathrm{t}}$, and we have an isomorphism $\left(\mathbf{C}_{\mathrm{t}}\right)_{+1} \cong \mathbf{C}$, which is identity on objects, and sends an arrow $f: X \rightarrow Y+I$ to $\triangleright_{1} \circ f: X \rightarrow Y$. 
Proof. Since coprojections are total, the coproduct diagram is in $\mathbf{C}_{\mathbf{t}}$. Let $f_{i}: X_{i} \rightarrow Y$ be total arrows. Then the cotuple $\left[f_{i}\right]_{i}: \coprod_{i} X_{i} \rightarrow Y$ is total, i.e. $\operatorname{Dp}\left(\left[f_{i}\right]_{i}\right)=1_{\coprod_{i} X_{i}}$ since $\operatorname{Dp}\left(\left[f_{i}\right]_{i}\right) \circ \kappa_{i}=\operatorname{Dp}\left(\left[f_{i}\right]_{i} \circ \kappa_{i}\right)=$ $\operatorname{Dp}\left(f_{i}\right)=1_{X_{i}}=\operatorname{Dp}\left(\kappa_{i}\right)=1_{\coprod_{i} X_{i}} \circ \kappa_{i}$ for all $i$. The object $I$ is final in $\mathbf{C}_{\mathrm{t}}$, because $\mathbf{C}_{\mathrm{t}}(X, I)=\left\{1_{X}\right\}$.

It is easy to see the mapping $f \mapsto \triangleright_{1} \circ f$ is functorial. To prove $\left(\mathbf{C}_{\mathrm{t}}\right)_{+1} \cong \mathbf{C}$, it suffices to show the functor is full and faithful. Let $f \in \mathbf{C}(X, Y)$. Using Lemma 4.8, we obtain $g: X \rightarrow Y+I$ by $g=$ $\kappa_{1} \circ f \otimes \kappa_{2} \circ \operatorname{Dp}(f)^{\perp}$. Then $g$ is total and $\triangleright_{1} \circ g=f$. Suppose that $h: X \rightarrow Y+I$ is a total arrow with $\triangleright_{1} \circ h=f$. Consider the decomposition $h=\kappa_{1} \circ h_{1} \otimes \kappa_{2} \circ h_{2}$, with $h_{1}=\triangleright_{1} \circ h$ and $h_{2}=\triangleright_{2} \circ h$. We have $h_{1}=f$ and $1=\operatorname{Dp}\left(h_{1}\right) \otimes \operatorname{Dp}\left(h_{2}\right)=\operatorname{Dp}(f) \otimes h_{2}$, so that $h_{2}=\operatorname{Dp}(f)^{\perp}$. Hence $h=g$.

Theorem 4.10. Let $(\mathbf{C}, I)$ be a FinPAC with effects. The subcategory $\mathbf{C}_{\mathrm{t}}$ is an effectus.

Proof. We have already seen that $\mathbf{C}_{\mathrm{t}}$ has finite coproducts $(+, 0)$ and a final object $I$. The joint monicity requirement is equivalent to say that $\triangleright_{1}, \triangleright_{2}: I+I \rightarrow I$ are jointly monic in $\left(\mathbf{C}_{\mathrm{t}}\right)_{+1}$, which is true since $\left(\mathbf{C}_{\mathrm{t}}\right)_{+1} \cong \mathbf{C}$. We prove that the squares $(\mathrm{E})$ and $\left(\mathrm{K}^{=}\right)$are pullbacks in $\mathbf{C}_{\mathrm{t}}$.

(E) Let $\alpha: Z \rightarrow A+Y$ and $\beta: Z \rightarrow B+X$ be total arrows with $(g+\mathrm{id}) \circ \alpha=($ id $+f) \circ \beta$. By postcomposing partial projections $\triangleright_{i}$ to $h:=(g+\mathrm{id}) \circ \alpha=(\mathrm{id}+f) \circ \beta$, we obtain $h_{1}=g \circ \alpha_{1}=\beta_{1}$ and $h_{2}=\alpha_{2}=f \circ \beta_{2}$, where $\alpha_{i}=\triangleright_{i} \circ \alpha, \beta_{i}=\triangleright_{i} \circ \beta$ and $h_{i}=\triangleright_{i} \circ h$. Note that $\operatorname{Dp}\left(\alpha_{1}\right)=\operatorname{Dp}\left(g \circ \alpha_{1}\right)=\operatorname{Dp}\left(h_{1}\right)$ and $\operatorname{Dp}\left(\beta_{2}\right)=\operatorname{Dp}\left(f \circ \beta_{2}\right)=\operatorname{Dp}\left(h_{2}\right)$. By Lemma 4.8, we can define a total arrow $\gamma: Z \rightarrow A+X$ by $\gamma=\kappa_{1} \circ \alpha_{1} \otimes \kappa_{2} \circ \beta_{2}$. As desired, $(\mathrm{id}+f) \circ \gamma=\kappa_{1} \circ \alpha_{1} \oslash \kappa_{2} \circ f \circ \beta_{2}=\kappa_{1} \circ \alpha_{1} \oslash \kappa_{2} \circ \alpha_{2}=\alpha$, and $(g+\mathrm{id}) \circ \gamma=\beta$ similarly. To see the uniqueness, assume that $\gamma: Z \rightarrow A+X$ satisfies $(\mathrm{id}+f) \circ \gamma=\alpha$ and $(g+\mathrm{id}) \circ \gamma=\beta$. Then one has $\triangleright_{1} \circ \gamma=\alpha_{1}$ and $\triangleright_{2} \circ \gamma=\alpha_{2}$. Hence the joint monicity of partial projections implies the uniqueness.

$\left(\mathbf{K}^{=}\right)$Let $\alpha: Z \rightarrow A$ and $\beta: Z \rightarrow A+X$ be total arrows with $\kappa_{1} \circ \alpha=(\mathrm{id}+f) \circ \beta$. By postcomposing partial projections $\triangleright_{i}$ to $\kappa_{1} \circ \alpha=(\mathrm{id}+f) \circ \beta$, we obtain $\alpha=\beta_{1}$ and $0=f \circ \beta_{2}$, where $\beta_{i}=\triangleright_{i} \circ \beta$. Then $\beta_{2}=0$ since $\operatorname{Dp}\left(\beta_{2}\right)=\operatorname{Dp}\left(f \circ \beta_{2}\right)=0$. Now we have $\beta=\kappa_{1} \circ \beta_{1} \oslash \kappa_{2} \circ \beta_{2}=\kappa_{1} \circ \alpha$ as desired.

Example 4.11. Recall from $\$ 2.2$ three examples of effectuses $\left(\text { Cstar }_{\mathrm{PU}}\right)^{\mathrm{op}}$, Set and $\mathcal{K} \ell(\mathcal{D})$. By Theorem 4.5, the Kleisli categories of the lift monads on these effectuses are FinPACs with effects (see also Table 1 in $\$ 1$ ).

The Kleisli category $\left(\left(\text { Cstar }_{\mathrm{PU}}\right)^{\mathrm{op}}\right)_{+1}$ is isomorphic to the opposite $\left(\text { Cstar }_{\mathrm{PSU}}\right)^{\text {op }}$ of the category of $C^{*}$-algebras and positive subunita $\mathrm{H}^{3}$ (PSU) maps. Indeed, we have the bijective correspondence shown on

$$
\frac{f: A \times \mathbb{C} \longrightarrow B \quad \text { PU-map }}{g: A \longrightarrow B \text { PSU-map }}
$$

the right, via $g(x)=f(x, 0)$ and $f(x, \lambda)=g(x)+\lambda(1-g(1))$. Predicates are PSU-maps $\mathbb{C} \rightarrow A$, which are easily identified with effects $p \in[0,1]_{A}:=\{p \in A \mid 0 \leq p \leq 1\}$. Then the domain predicate of a PSUmap $f: A \rightarrow B$ is identified with $f(1) \in[0,1]_{B}$. By Lemma 4.7 2, the sum $f \otimes g$ of PSU-maps $f, g: A \rightarrow B$ is defined precisely when $f(1) \perp g(1)$ in $[0,1]_{B}$, namely $f(1)+g(1) \leq 1$. In that case the sum is defined pointwise: $(f \otimes g)(x)=f(x)+g(x)$. Note that $C^{*}$-algebras with completely positive subunital maps and $W^{*}$-algebras with normal (completely) positive subunital maps work in exactly the same way. The latter is especially important for semantics of quantum programming languages [2,11].

For the classical example, it is well-known that $\mathbf{S e t}_{+1} \cong \mathbf{P f n}$, where $\mathbf{P f n}$ is the category of sets and partial functions. The domain predicate $\operatorname{Dp}(f)$ of a partial function $f: X \rightarrow Y$ is identified with its domain of definition $\operatorname{dom}(f) \subseteq X$. The sum of $f, g: X \rightarrow Y$ is defined precisely if $\operatorname{dom}(f) \perp \operatorname{dom}(g)$ in $\mathcal{P}(X)$, i.e. $\operatorname{dom}(f) \cap \operatorname{dom}(g)=\varnothing$. In that case $f \otimes g$ is defined on $\operatorname{dom}(f) \cup \operatorname{dom}(g)$ in an obvious way.

For the probabilistic example, we have $\mathcal{K} \ell(\mathcal{D})_{+1} \cong \mathcal{K} \ell(\widehat{\mathcal{D}})$, where $\widehat{\mathcal{D}}=\widehat{\mathcal{D}}_{[0,1]}$ is the subdistribution monad over $[0,1]$. This is due to the natural bijections $\widehat{\mathcal{D}}(X) \cong \mathcal{D}(X+1)$. The domain predicate $\operatorname{Dp}(f)$ of a map $f: X \rightarrow Y$ in $\mathcal{K} \ell(\widehat{\mathcal{D}})$, i.e. a function $f: X \rightarrow \widehat{\mathcal{D}}(Y)$ is identified with the 'fuzzy' predicate

\footnotetext{
${ }^{3}$ A positive map $g: A \rightarrow B$ is said to be subunital if $g(1) \leq 1$.
} 
$p \in[0,1]^{X}$ given by $p(x)=\sum_{y} f(x)(y)$. The sum of functions $f, g: X \rightarrow \widehat{\mathcal{D}} Y$ is defined if and only if $\sum_{y} f(-)(y) \perp \sum_{y} g(-)(y)$ in $[0,1]^{X}$, that is, $\sum_{y} f(x)(y)+\sum_{y} g(x)(y) \leq 1$ for all $x \in X$. In that case, the sum is defined by $(f \otimes g)(x)(y)=f(x)(y)+g(x)(y)$.

\section{Categorical equivalence of effectuses and FinPACs with effects}

The results in the previous section are summarised as follows. For an effectus $\mathbf{B}$, the category $\mathbf{B}_{+1}$ with $1 \in \mathbf{B}_{+1}$ is a FinPAC with effects; and for a FinPAC with effects $(\mathbf{C}, I)$, the subcategory $\mathbf{C}_{t}$ is an effectus. Moreover we have isomorphisms $\mathbf{B} \cong\left(\mathbf{B}_{+1}\right)_{\mathrm{t}}$ and $\mathbf{C} \cong\left(\mathbf{C}_{\mathrm{t}}\right)_{+1}$. We can immediately obtain a characterisation of effectuses.

Corollary 5.1. A category $\mathbf{B}$ is an effectus if and only if there is a FinPAC with effects $(\mathbf{C}, I)$ such that $\mathbf{B} \cong \mathbf{C}_{\mathrm{t}}$.

The results are most naturally presented in terms of (2-)categorical equivalence.

Definition 5.2. We define a (strict) 2-category Eff of effectuses as follows. An object is an effectus $\mathbf{B}$. An arrow $F: \mathbf{A} \rightarrow \mathbf{B}$ is a functor that preserves the final object and finite coproducts. A 2-cell $\alpha: F \Rightarrow G$ is a natural transformation that is monoidal w.r.t. $(+, 0)$. We also define a 2-category FPE of FinPACs with effects as follows. An object is a FinPAC with effects $(\mathbf{C}, I)$. An arrow $F:\left(\mathbf{C}, I_{\mathbf{C}}\right) \rightarrow\left(\mathbf{D}, I_{\mathbf{D}}\right)$ is a functor $F: \mathbf{C} \rightarrow \mathbf{D}$ that preserves finite coproducts and "preserves the truth" in the sense that $1_{F I_{\mathbf{C}}}: F I_{\mathbf{C}} \rightarrow$ $I_{\mathbf{D}}$ is an isomorphism, and $1_{F I} \circ F 1_{X}=1_{F X}$ for all $X \in \mathbf{C}$. A 2-cell $\alpha: F \Rightarrow G$ is a natural transformation that is monoidal w.r.t. $(+, 0)$, and satisfies $1_{G I} \circ \alpha_{I}=1_{F I}$.

Theorem 5.3. The assignments $\mathbf{B} \mapsto \mathbf{B}_{+1}$ and $\mathbf{C} \mapsto \mathbf{C}_{\mathrm{t}}$ extend to 2 -functors $(-)_{+1}$ : Eff $\rightarrow \mathbf{F P E}$ and $(-)_{\mathrm{t}}: \mathbf{F P E} \rightarrow \mathbf{E f f}$ respectively. Moreover, they form a 2-equivalence of 2-categories $\mathbf{E f f} \simeq \mathbf{F P E}$.

Proof. The essential part is already done. The rest, checking functoriality and naturality, is mostly routine. We defer the details to Appendix $B$.

\section{State-and-effect triangles over FinPACs with effects}

Let $(\mathbf{C}, I)$ be a FinPAC with effects. Recall that $\operatorname{Pred}(X)=\mathbf{C}(X, I)$ is the set of predicates on $X$. We call an arrow $\omega: I \rightarrow X$ a substate on $X$, an arrow $r: I \rightarrow I$ a scalar. We write $S \operatorname{Stat}(X)=\mathbf{C}(I, X)$ for the set of substates on $X$, and let $M=\mathbf{C}(I, I)$ be the set of scalars.

Proposition 6.1. Let $(\mathbf{C}, I)$ be a FinPAC with effects.

1. The effect algebra $M=\mathbf{C}(I, I)$ is an effect monoid with the composition $\circ$ as a multiplication.

2. For each $X \in \mathbf{C}$, the effect algebra Pred $(X)=\mathbf{C}(X, I)$ is an effect module over $M$, with the composition $\circ$ as a scalar multiplication.

3. For each $X \in \mathbf{C}$, the PCM SStat $(X)=\mathbf{C}(I, X)$ is a PCMod over $M$ with the composition $\circ$ as a (right) scalar multiplication. Moreover it is subconvex.

Proof. Straightforward, but note that $1_{I}=\mathrm{id}_{I} \in M$. To see $S \operatorname{Stat}(X)$ is subconvex, use $\operatorname{Dp}(\omega \circ r) \leq r$.

Combining the dual adjunction $\left(\mathbf{G E M o d}_{M}\right)^{\text {op }} \rightleftarrows \mathbf{S C o n v}_{M}$ from Proposition 2.1, we obtain a stateand-effect triangle. We use the category $\mathbf{G E M o d}_{M}$ of GEMod's because induced predicate transformers do not necessarily preserve the truth predicates. 
Theorem 6.2. For a FinPAC with effects $(\mathbf{C}, I)$, the hom-functors $\mathbf{C}(-, I)$ and $\mathbf{C}(I,-)$ give rise to the functors in the diagram on the right, constituting a state-and-effect triangle.

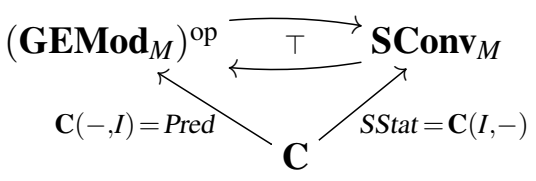

Proof. It is easy to check that the precomposition $\mathbf{C}(f, I)$ and the postcomposition $\mathbf{C}(I, f)$ are desired homomorphisms.

Examples of this type of state-and-effect triangles have already appeared in [4, 10], but the general construction is new. Substates in the quantum example $\left(\text { Cstar }_{\text {PSU }}\right)^{\text {op }}$ are PSU-maps $\omega: A \rightarrow \mathbb{C}$. In the classical example Pfn, substates on $X$ are either elements $x \in X$ or the 'bottom'. In the probabilistic example $\mathcal{K} \ell(\widehat{\mathcal{D}})$, substates are subdistributions $\omega \in \widehat{\mathcal{D}}(X)$.

As is the case for effectuses $(\$ 2.2$, there is an abstract Born rule given by $(\omega \vDash p):=p \circ \omega \in M$ for $\omega: I \rightarrow X$ and $p: X \rightarrow I$. The map $\vDash: \operatorname{SStat}(X) \times \operatorname{Pred}(X) \rightarrow M$ is an appropriate bihomomorphism, so that by "currying", we obtain the following maps $\alpha_{X}$ and $\beta_{X}$ in the bijective correspondence of the dual adjunction.

$$
\frac{\alpha_{X}: \operatorname{Pred}(X) \longrightarrow \operatorname{SConv}_{M}(\operatorname{SStat}(X), M) \text { in } \operatorname{GEMod}_{M}}{\beta_{X}: \operatorname{SStat}(X) \longrightarrow \operatorname{GEMod}_{M}(\operatorname{Pred}(X), M) \text { in } \operatorname{SConv}_{M}}
$$

These maps $\alpha$ and $\beta$ give natural transformations which fill the state-and-effect triangle

In a FinPAC with effects, a state on $X$ is a substate $\omega: I \rightarrow X$ with $\operatorname{Dp}(\omega)=1$ (i.e. a total substate), and the set of states is denoted by $\operatorname{Stat}(X)=\mathbf{C}_{\mathrm{t}}(I, X)$. This definition accords with states in an effectus, since $\left(\mathbf{B}_{+1}\right)_{\mathrm{t}}(1, X) \cong \mathbf{B}(1, X)$. The set $\operatorname{Stat}(X)$ is a subset of $\operatorname{SStat}(X)$ that is closed under convex sum, hence $\operatorname{Stat}(X)$ is a convex set, giving a functor $S t a t: \mathbf{C}_{t} \rightarrow \operatorname{Conv}_{M}$. On the other hand, we obtain a functor Pred: $\mathbf{C}_{\mathrm{t}} \rightarrow\left(\mathbf{E M o d}_{M}\right)^{\mathrm{op}}$ as a restriction of Pred: $\mathbf{C} \rightarrow\left(\operatorname{GEMod}_{M}\right)^{\mathrm{op}}$, since predicate transformers induced by total arrows preserve the truth predicates. This is an alternative way to obtain a state-and-effect triangle over an effectus shown in Figure 1] (cf. [5]).

In what follows, we will focus on a FinPAC with effects satisfying 'normalisation' (of states). A FinPAC with effects $(\mathbf{C}, I)$ satisfies normalisation if for each object $X$ and for each substate $\omega \in S S t a t(X)$ that is nonzero $\left(\omega \neq 0_{I X}\right)$, there exists a unique state $\tilde{\omega} \in \operatorname{Stat}(X)$ such that $\omega=\tilde{\omega} \circ \operatorname{Dp}(\omega)$. An effectus $\mathbf{B}$ satisfies normalisation if the corresponding FinPAC with effects $\left(\mathbf{B}_{+1}, 1\right)$ satisfies normalisation. An effectus with normalisation was introduced and studied in [7], where most results are restricted to the case when the set of scalars $M$ is the unit interval [0,1]. In fact, if an effectus or FinPAC with effects satisfies normalisation, then the scalars are already 'good' enough to take away the restriction $M=[0,1]$.

Definition 6.3. An effect monoid $M$ has division if for all $s, t \in M$ with $s \leq t$ and $t \neq 0$, there exists unique 'quotient' $q \in M$ such that $q \cdot t=s$. The quotient $q$ is denoted by $s / t$. We call such an effect monoid a division effect monoid.

Proposition 6.4. If a FinPAC with effects $(\mathbf{C}, I)$ satisfies normalisation, then the effect monoid of scalars $M=\mathbf{C}(I, I)$ has division.

Proof. Let $s, t \in M$ be scalars with $s \leq t$ and $t \neq 0$. Let $s^{\prime}=t \ominus s$, so that $s \oslash s^{\prime}=t$. Let $\omega=\kappa_{1} \circ$ $s \otimes \kappa_{2} \circ s^{\prime}: I \rightarrow I+I$, which is nonzero because $\operatorname{Dp}(\omega)=s \otimes s^{\prime}=t \neq 0$. By normalisation there is a state $\tilde{\omega}: I \rightarrow I+I$ with $\omega=\tilde{\omega} \circ \operatorname{Dp}(\omega)=\tilde{\omega} \circ t$. Then $s=\triangleright_{1} \circ \omega=\triangleright_{1} \circ \tilde{\omega} \circ t$. Therefore $\triangleright_{1} \circ \tilde{\omega}$ is a desired quotient. To see the uniqueness of the quotient, assume that $q \in M$ satisfies $s=q \circ t$. Then $s^{\prime}=t \ominus s=t \ominus(q \circ t)=q^{\perp} \circ t$. Let $\omega_{q}=\kappa_{1} \circ q \otimes \kappa_{2} \circ q^{\perp}: I \rightarrow I+I$, which is a state and $\omega_{q} \circ t=$ $\kappa_{1} \circ q \circ t \otimes \kappa_{2} \circ q^{\perp} \circ t=\kappa_{1} \circ s \otimes \kappa_{2} \circ s^{\prime}=\omega$. By the uniqueness of normalisation, we obtain $\omega_{q}=\tilde{\omega}$. Therefore $\triangleright_{1} \circ \tilde{\omega}=\triangleright_{1} \circ \omega_{q}=q$. 
The division indeed satisfies desired properties, see Lemmas C.1 and C.2. It allows us to obtain the following result, by generalising $M=[0,1]$ to any division effect monoid.

Theorem 6.5 ([7, Corollary 19]). Let $\mathbf{B}$ be an effectus satisfying normalisation. Then, all the categories and the functors in the state-and-effect triangle over $\mathbf{B}$ (Figure 1) are objects and arrows in $\mathbf{E f f}$.

Note that, unlike [7], we simply use $\operatorname{Conv}_{M}$ rather than the category of cancellative convex sets. This is because we use a weaker variant of the joint monicity requirement in Definition 2.2, and $\mathbf{C o n v}_{M}$ is indeed an effectus in our sense; see Proposition C.3. Furthermore, it is straightforward to check the following.

Lemma 6.6. Let $M$ be a division effect monoid. The unit and the counit of the adjunction $\left(\mathbf{E M o d}_{M}\right)^{\mathrm{op}} \rightleftarrows$ $\mathbf{C o n v}_{M}$ are 2-cells in Eff. Namely, $\left(\mathbf{E M o d}_{M}\right)^{\mathrm{op}} \rightleftarrows \mathbf{C o n v}_{M}$ is an adjunction in the 2-category Eff.

In the light of the 2-equivalence $\mathbf{E f f} \simeq \mathbf{F P E}$, we obtain a corresponding state-and-effect triangle over a FinPAC with effects.

Corollary 6.7. Let $(\mathbf{C}, I)$ be a FinPAC with effects satisfying normalisation. We have a state-and-effect triangle on the right, where the categories, the functors and the adjunction are in $\mathbf{F P E}$.

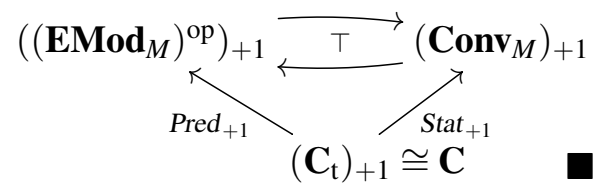

\section{Conclusions}

We studied partial computation in effectuses, giving a fundamental equivalence of effectuses and FinPACs with effects. Despite the equivalence, FinPACs with effects sometimes have an advantage over effectuses, because they have richer structures such as the finitely partially additive structure. For instance, an instrument map instr $p: X \rightarrow X+\cdots+X$ for an ' $n$-test' $p: X \rightarrow 1+\cdots+1$ in an effectus allow us to perform a (quantum) measurement, with $n$ outcomes [5, Assumption 2]. Switching to a FinPAC with effects, we can decompose such an instrument map to $n$ 'partial' endomaps $X \rightarrow X$, which give a simpler formulation. The details will be elaborated in a subsequent paper.

Recently the author and his colleagues studied quotient-comprehension chains [3] which are related to such instrument maps and measurement. It is worth noting that many examples of quotientcomprehension chains are given by FinPACs with effects, including a quantum setting via $W^{*}$-algebras. An important future work is thus to give a categorical axiomatisation of such a quotient-comprehension chain in the effectus / 'FinPAC with effects' framework.

\section{Acknowledgements}

I would like to thank Bart Jacobs for suggesting the problem to characterise the Kleisli category of the lift monad on an effectus; Bas Westerbaan for fruitful discussions to solve it; and Robin Adams for helpful comments.

\section{References}

[1] Michael A. Arbib \& Ernest G. Manes (1980): Partially additive categories and flow-diagram semantics. Journal of Algebra 62(1), pp. 203-227, doi:10.1016/0021-8693(80)90212-4.

[2] Kenta Cho (2014): Semantics for a Quantum Programming Language by Operator Algebras. In: QPL 2014, EPTCS 172, pp. 165-190, doi 10.4204/EPTCS.172.12. 
[3] Kenta Cho, Bart Jacobs, Bas Westerbaan \& Bram Westerbaan (2015): Quotient-Comprehension Chains. In: QPL 2015. To appear.

[4] Bart Jacobs (2014): Dijkstra Monads in Monadic Computation. In: CMCS 2014, LNCS 8446, Springer, pp. 135-150, doi:10.1007/978-3-662-44124-4_8

[5] Bart Jacobs (2015): New Directions in Categorical Logic, for Classical, Probabilistic and Quantum Logic. LMCS. To appear. arXiv:1205.3940v4 [math.LO].

[6] Bart Jacobs \& Jorik Mandemaker (2012): Coreflections in Algebraic Quantum Logic. Foundations of Physics 42(7), pp. 932-958, doi:10.1007/s10701-012-9654-8.

[7] Bart Jacobs, Bas Westerbaan \& Bram Westerbaan (2015): States of Convex Sets. In: FoSSaCS 2015, LNCS 9034, Springer, pp. 87-101, doi:10.1007/978-3-662-46678-0_6

[8] Ernest G. Manes \& Michael A. Arbib (1986): Algebraic Approaches to Program Semantics. Monographs in Computer Science, Springer, doi:10.1007/978-1-4612-4962-7

[9] Eugenio Moggi (1991): Notions of computation and monads. Information and Computation 93(1), pp. 55-92, doi:10.1016/0890-5401(91)90052-4.

[10] Mathys Rennela (2013): On operator algebras in quantum computation. Master's thesis, Université Paris 7 Denis Diderot.

[11] Mathys Rennela (2014): Towards a Quantum Domain Theory: Order-enrichment and Fixpoints in $W^{*}$ algebras. In: MFPS XXX, ENTCS 308, Elsevier, pp. 289-307, doi 10.1016/j.entcs.2014.10.016

\section{A Omitted proofs in Section 3}

We write $[n]=\{1, \ldots, n\}$ for the $n$ element set, and $n \cdot X=\coprod_{i \in[n]} X$ for an $n$-fold coproduct.

Lemma A.1. In a FinPAC the following hold.

1. A family $\left(f_{i}: X \rightarrow Y\right)_{i \in[n]}$ is orthogonal whenever $\left(f_{i}\right)_{i \in[n]}$ is compatible in the sense that there exists a 'bound' $b: X \rightarrow n \cdot Y$ such that $f_{i}=\triangleright_{i} \circ b$.

2. If a family $\left(f_{i}: X \rightarrow Y\right)_{i \in[n]}$ is orthogonal, then a family $\left(\kappa_{i} \circ f_{i}: X \rightarrow n \cdot Y\right)_{i \in[n]}$ is orthogonal too.

Proof. 1. We prove the following stronger statement by induction on $n$.

- If a family $\left(f_{i}: X \rightarrow Y\right)_{i \in[n]}$ is compatible via a bound $b: X \rightarrow n \cdot Y$, then it is orthogonal and $\bigotimes_{i \in[n]} f_{i}=\nabla \circ b$.

The base case $(n=0)$ is trivial. To show the induction step, let $\left(f_{i}: X \rightarrow Y\right)_{i \in[n+1]}$ be a compatible family via a bound $b: X \rightarrow(n+1) \cdot Y$. Let $\alpha:(n+1) \cdot Y \rightarrow n \cdot Y+Y$ be the canonical associativity isomorphism. Then it is easy to see that $\left(f_{i}\right)_{i \in[n]}$ is compatible via $\triangleright_{1} \circ \alpha \circ b: X \rightarrow n \cdot Y$. By the induction hypothesis $\left(f_{i}\right)_{i \in[n]}$ is orthogonal and $\bigotimes_{i \in[n]} f_{i}=\nabla \circ \triangleright_{1} \circ \alpha \circ b$. Note that

$$
\begin{aligned}
& \triangleright_{1} \circ(\nabla+\mathrm{id}) \circ \alpha \circ b=\nabla \circ \triangleright_{1} \circ \alpha \circ b=\bigotimes_{i \in[n]} f_{i} \\
& \triangleright_{2} \circ(\nabla+\mathrm{id}) \circ \alpha \circ b=\triangleright_{2} \circ \alpha \circ b=\triangleright_{n+1} \circ b=f_{n+1} .
\end{aligned}
$$

Therefore $\bigotimes_{i \in[n]} f_{i} \perp f_{n+1}$ via $(\nabla+\mathrm{id}) \circ \alpha \circ b$, so that $\left(f_{i}\right)_{i \in[n+1]}$ is orthogonal. Moreover we have

$$
\bigotimes_{i \in[n+1]} f_{i}=\left(\bigotimes_{i \in[n]} f_{i}\right) \otimes f_{n+1}=\nabla \circ(\nabla+\mathrm{id}) \circ \alpha \circ b=\nabla \circ b
$$


2. We prove it by induction on $n$. The base case $n=0$ is trivial. Let $\left(f_{i}: X \rightarrow Y\right)_{i \in[n+1]}$ be an orthogonal family. Then $n$ arrows $f_{1} \otimes f_{n+1}, f_{2}, \ldots, f_{n}$ are orthogonal. By the induction hypothesis,

$$
\kappa_{1} \circ\left(f_{1} \oslash f_{n+1}\right)=\kappa_{1} \circ f_{1} \otimes \kappa_{1} \circ f_{n+1}, \kappa_{2} \circ f_{2}, \ldots, \kappa_{n} \circ f_{n}: X \rightarrow n \cdot Y
$$

are orthogonal. This implies that $\emptyset_{i \in[n]} \kappa_{i} \circ f_{i}$ and $\kappa_{1} \circ f_{n+1}$ are orthogonal. By the untying axiom, $\kappa_{1} \circ \emptyset_{i \in[n]} \kappa_{i} \circ f_{i}=\emptyset_{i \in[n]} \kappa_{1} \circ \kappa_{i} \circ f_{i}$ and $\kappa_{2} \circ \kappa_{1} \circ f_{n+1}$ are orthogonal. It follows that

$$
\kappa_{1} \circ \kappa_{1} \circ f_{1}, \ldots, \kappa_{1} \circ \kappa_{n} \circ f_{n}, \kappa_{2} \circ \kappa_{1} \circ f_{n+1}: X \rightarrow n \cdot Y+n \cdot Y
$$

are orthogonal. Let $\alpha: n \cdot Y+Y \rightarrow(n+1) \cdot Y$ be the associativity isomorphism. Then

$$
\begin{aligned}
\alpha \circ\left(\mathrm{id}+\triangleright_{1}\right) \circ \kappa_{1} \circ \kappa_{i} \circ f_{i} & =\alpha \circ \kappa_{1} \circ \kappa_{i} \circ f_{i}=\kappa_{i} \circ f_{i} \\
\alpha \circ\left(\mathrm{id}+\triangleright_{1}\right) \circ \kappa_{2} \circ \kappa_{1} \circ f_{n+1} & =\alpha \circ \kappa_{2} \circ \triangleright_{1} \circ \kappa_{1} \circ f_{n+1}=\kappa_{n+1} \circ f_{n+1}
\end{aligned}
$$

Therefore $\left(\kappa_{i} \circ f_{i}: X \rightarrow(n+1) \cdot Y\right)_{i \in[n+1]}$ is orthogonal.

Proof of Theorem 3.4 (Only if) In a FinPAC, the partial projections are jointly monic by Lemma 3.3 To show the pullback condition, let $f, g: Y \rightarrow X+X$ be arrows with $\nabla \circ f=\triangleright_{1} \circ g$. Let $f_{i}=\triangleright_{i} \circ f$ and $g_{i}=\triangleright_{i} \circ g(i=1,2)$. Using Lemma 3.2, one has $f_{1} \perp f_{2}, g_{1} \perp g_{2}$, and $f_{1} \otimes f_{2}=\nabla \circ f=\triangleright_{1} \circ g=g_{1}$, so that $f_{1}, f_{2}, g_{2}$ are orthogonal. By (ternary) untying, $\kappa_{1} \circ f_{1}, \kappa_{2} \circ f_{2}, \kappa_{3} \circ g_{2}: Y \rightarrow X+X+X$ are orthogonal. Writing $\alpha: X+X+X \rightarrow(X+X)+X$ for the associativity isomorphism, define $h: Y \rightarrow(X+X)+X$ by

$$
\begin{aligned}
h & =\alpha \circ\left(\kappa_{1} \circ f_{1} \oslash \kappa_{2} \circ f_{2} \oslash \kappa_{3} \circ g_{2}\right) \\
& =\kappa_{1} \circ \kappa_{1} \circ f_{1} \oslash \kappa_{1} \circ \kappa_{2} \circ f_{2} \oslash \kappa_{2} \circ g_{2} \\
& \left.=\kappa_{1} \circ f \oslash \kappa_{2} \circ g_{2} \quad \text { (note } f=\kappa_{1} \circ f_{1} \oslash \kappa_{2} \circ f_{2}\right) .
\end{aligned}
$$

Then $\triangleright_{1} \circ h=f$ easily, and

$$
(\nabla+\mathrm{id}) \circ h=\kappa_{1} \circ \nabla \circ f \otimes \kappa_{2} \circ g_{2}=\kappa_{1} \circ g_{1} \oslash \kappa_{2} \circ g_{2}=g
$$

Hence $h$ is a desired mediating map. To see the uniqueness, let $k: Y \rightarrow(X+X)+X$ be an arrow with $\triangleright_{1} \circ k=f$ and $(\nabla+\mathrm{id}) \circ k=g$. Then $\triangleright_{2} \circ k=\triangleright_{2} \circ(\nabla+\mathrm{id}) \circ k=\triangleright_{2} \circ g$. Such $k$ is unique since the partial projections $\triangleright_{1}$ and $\triangleright_{2}$ are jointly monic.

(If) Assume that a category $\mathbf{C}$ satisfies the given conditions. The joint monicity of partial projections allows us to define the partial sum $\oslash$ on homsets $\mathbf{C}(X, Y)$ in the way of Proposition 3.2. We show that $\mathbf{C}$ is PCM-enriched with zero arrows as neutral elements.

Associativity. Let $f, g, h \in \mathbf{C}(X, Y)$ be arrows with $f \perp g$ (i.e. compatible) via $b: X \rightarrow Y+Y$, and $f \otimes g \perp h$ via $c: X \rightarrow Y+Y$. By definition we have $\nabla \circ b=f \otimes g=\triangleright_{1} \circ c$, so that we obtain a mediating map $d$ as in the diagram:

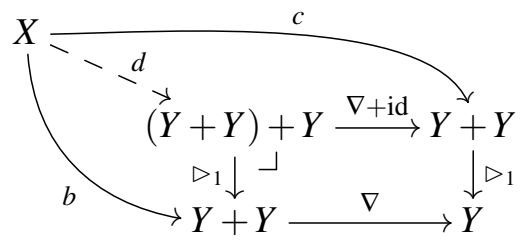

Then, it is straightforward to check that

$$
g \perp h \text { via } X \stackrel{d}{\rightarrow}(Y+Y)+Y \stackrel{\triangleright_{2}+\mathrm{id}}{\longrightarrow} Y+Y \quad ; \text { and } \quad f \perp g \otimes h \text { via } X \stackrel{d}{\rightarrow}(Y+Y)+Y \stackrel{\left[\mathrm{id}, \kappa_{2}\right]}{\longrightarrow} Y+Y .
$$


Finally we have

$$
f \otimes(g \otimes h)=\nabla \circ\left[\mathrm{id}, \kappa_{2}\right] \circ d=[\nabla, \mathrm{id}] \circ d=\nabla \circ(\nabla+\mathrm{id}) \circ d=\nabla \circ c=(f \otimes g) \otimes h .
$$

Commutativity. Let $f, g \in \mathbf{C}(X, Y)$ be arrows with $f \perp g$ via $b: X \rightarrow Y+Y$. Then it is easy to see that $g \perp f$ via $\left[\kappa_{2}, \kappa_{1}\right] \circ b: X \rightarrow Y+Y$, and that $g \otimes f=\nabla \circ\left[\kappa_{2}, \kappa_{1}\right] \circ b=\nabla \circ b=f \otimes g$.

Zero. For $f \in \mathbf{C}(X, Y)$, we have $0_{X Y} \perp f$ via $\kappa_{2} \circ f: X \rightarrow Y+Y$, and $0_{X Y} \oslash f=\nabla \circ \kappa_{2} \circ f=f$.

Therefore $\mathbf{C}(X, Y)$ is a PCM for each $X, Y \in \mathbf{C}$. We need to show that the composition $\circ: \mathbf{C}(Y, Z) \times$ $\mathbf{C}(X, Y) \rightarrow \mathbf{C}(X, Z)$ is a PCM-bihomomorphism. Let $f \in \mathbf{C}(X, Y)$ and $h, k \in \mathbf{C}(Y, Z)$ be arrows with $h \perp k$ via $b: Y \rightarrow Z+Z$. Then $h \circ f \perp k \circ f$ via $b \circ f: X \rightarrow Z+Z$, and $h \circ f \otimes k \circ f=\nabla \circ b \circ f=(h \otimes k) \circ f$. We also have $0 \circ f=0$. Hence $(-) \circ f$ is a PCM-homomorphism. Next, let $h \in \mathbf{C}(Y, Z)$ and $f, g \in$ $\mathbf{C}(X, Y)$ be arrows with $f \perp g$ via $b: X \rightarrow Y+Y$. Then $h \circ f \perp h \circ g$ via $(h+h) \circ b: X \rightarrow Z+Z$, and $h \circ f \otimes h \circ f=\nabla \circ(h+h) \circ b=h \circ \nabla \circ b=h \circ(f \otimes g)$. We also have $h \circ 0=0$, and hence $h \circ(-)$ is a PCM-homomorphism.

We have shown that $\mathbf{C}$ is PCM-enriched. The compatibility sum axiom holds by definition. If $f, g: X \rightarrow Y$ are compatible (i.e. orthogonal) via $b: X \rightarrow Y+Y$, then $\kappa_{1} \circ f, \kappa_{2} \circ g: X \rightarrow Y+Y$ are compatible via $\left(\kappa_{1}+\kappa_{2}\right) \circ b: X \rightarrow(Y+Y)+(Y+Y)$. Hence the untying axiom holds.

\section{B Proof of a 2-equivalence of the 2-categories of effectuses and FinPACs with effects}

Note first that, by definition, a natural transformation $\alpha: F \rightarrow G$ is monoidal w.r.t. $(+, 0)$ if the following diagrams commute.

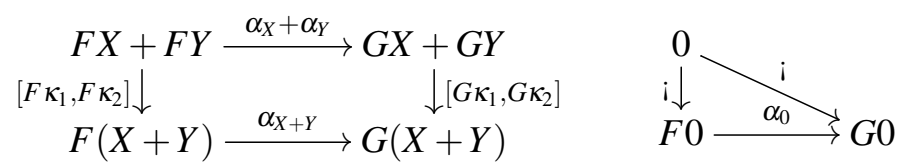

Obviously, the right-hand diagram always commutes. Hence $\alpha$ is $(+, 0)$-monoidal if and only if the left-hand diagram commutes, i.e. when $\alpha_{X+Y} \circ F \kappa_{1}=G \kappa_{1} \circ \alpha_{X}$ and $\alpha_{X+Y} \circ F \kappa_{2}=G \kappa_{2} \circ \alpha_{Y}$.

Let $F: \mathbf{A} \rightarrow \mathbf{B}$ be a functor between effectuses in $\mathbf{E f f}$, i.e. a functor that preserves 1 and $(0,+)$. Then, the canonical arrow $F X+1 \rightarrow F(X+1)$ in the diagram below is an isomorphism.

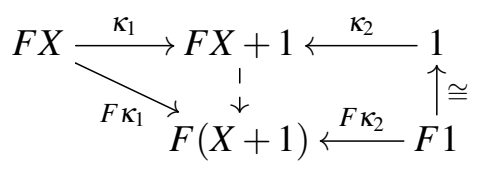

We denote the inverse $F(X+1) \rightarrow F X+1$ by $l_{F, X}$ or simply by $l_{X}$. We then have the following equations, which will be used repeatedly.

$$
\begin{aligned}
& l_{F, X} \circ F \kappa_{1}=\kappa_{1} \\
& l_{F, X} \circ F \kappa_{2}=\kappa_{2} \circ !_{F 1}
\end{aligned}
$$

Lemma B.1. Let $F: \mathbf{A} \rightarrow \mathbf{B}$ be a functor between effectuses in $\mathbf{E f f}$. Then we have a functor $F_{+1}: \mathbf{A}_{+1} \rightarrow$ $\mathbf{B}_{+1}$ which is a 'lifting' of $F$ in the sense that the following diagram commutes.

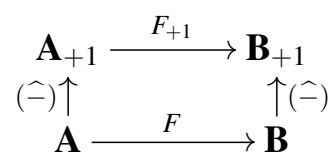


Proof. We define $F_{+1}$ by $F_{+1} X=F X$ and $F_{+1}(f)=l_{F, Y} \circ F f: F X \rightarrow F Y+1$ for $f: X \rightarrow Y$ in $\mathbf{A}_{+1}$, i.e. $f: X \rightarrow Y+1$ in $\mathbf{A}$. For $h: X \rightarrow Y$ in $\mathbf{A}$, using (2),

$$
F_{+1} \widehat{h}=l_{Y} \circ F \kappa_{1} \circ F h=\kappa_{1} \circ F h=\widehat{F h} .
$$

Therefore $F_{+1}$ is a lifting of $F$. Taking $h=\operatorname{id}_{X}$ we obtain $F_{+1}\left(\widehat{\mathrm{id}}_{X}\right)=\widehat{\mathrm{id}}_{F X}$. Let $f: X \rightarrow Y$ and $g: Y \rightarrow Z$ be arrows in $\mathbf{A}_{+1}$. Note that we have

$$
\begin{aligned}
& l_{Z} \circ F\left[g, \kappa_{2}\right] \circ F \kappa_{1}=l_{Z} \circ F g \\
& =\left[l_{Z} \circ F g, \kappa_{2}\right] \circ \kappa_{1} \\
& =\left[l_{Z} \circ F g, \kappa_{2}\right] \circ l_{Y} \circ F \kappa_{1} \quad \text { by (2) } \\
& l_{Z} \circ F\left[g, \kappa_{2}\right] \circ F \kappa_{2}=l_{Z} \circ F \kappa_{2} \\
& =\kappa_{2} \circ !_{F 1} \quad \text { by (3) } \\
& =\left[l_{Z} \circ F g, \kappa_{2}\right] \circ \kappa_{2} \circ !_{F 1} \\
& =\left[l_{Z} \circ F g, \kappa_{2}\right] \circ l_{Y} \circ F \kappa_{2} \quad \text { by (3). }
\end{aligned}
$$

Because $F$ preserves finite coproducts and hence $F Y \stackrel{F \kappa_{1}}{\longrightarrow} F(Y+1) \stackrel{F \kappa_{2}}{\longleftarrow} F 1$ is a coproduct in $\mathbf{B}$, we obtain $l_{Z} \circ F\left[g, \kappa_{2}\right]=\left[l_{Z} \circ F g, \kappa_{2}\right] \circ l_{Y}$. Then,

$$
F_{+1}(g \hat{\circ} f)=l_{Z} \circ F\left[g, \kappa_{2}\right] \circ F f=\left[l_{Z} \circ F g, \kappa_{2}\right] \circ l_{Y} \circ F f=F_{+1} g \hat{\circ} F_{+1} f .
$$

Therefore $F_{+1}$ is a functor.

Lemma B.2. The mapping $\mathbf{B} \mapsto\left(\mathbf{B}_{+1}, 1\right)$ for an effectus $\mathbf{B}$ gives rise to a 2 -functor $(-)_{+1}: \mathbf{E f f} \rightarrow \mathbf{F P E}$.

Proof. Recall $\left(\mathbf{B}_{+1}, 1\right)$ is a FinPAC with effects by Theorem 4.5. For an arrow $F: \mathbf{A} \rightarrow \mathbf{B}$ in Eff, we have a functor $F_{+1}: \mathbf{A}_{+1} \rightarrow \mathbf{B}_{+1}$ by Lemma B.1. Since $F_{+1}$ is a lifting of $F$, the functor $F_{+1}$ preserves finite coproducts as $F$ does. The arrow $1_{F 1}=!_{F 1}: F 1 \rightarrow 1$ is an isomorphism because $!_{F 1}: F 1 \rightarrow 1$ is an isomorphism. Since $F_{+1} 1_{X}=F_{+1} \widehat{!}_{X}=\widehat{F !_{X}}$ is total, we have $1_{F 1} \hat{\circ} F_{+1} 1_{X}=1_{F X}$. Therefore $F_{+1}$ is an arrow in FPE.

Let $F, G: \mathbf{A} \rightarrow \mathbf{B}$ be arrows and $\alpha: F \Rightarrow G$ a 2-cell in Eff. Note the equation

$$
\left(\alpha_{X}+\mathrm{id}_{1}\right) \circ l_{F, X}=l_{G, X} \circ \alpha_{X+1}
$$

which holds because

$$
\begin{aligned}
\left(\alpha_{X}+\mathrm{id}\right) \circ l_{F, X} \circ F \kappa_{1} & =\left(\alpha_{X}+\mathrm{id}\right) \circ \kappa_{1} & & \text { by (2) } \\
& =\kappa_{1} \circ \alpha_{X} & & \\
& =l_{G, X} \circ G \kappa_{1} \circ \alpha_{X} & & \text { by (2) } \\
& =l_{G, X} \circ \alpha_{X+1} \circ F \kappa_{1} & & \text { since } \alpha \text { is }(+, 0) \text {-monoidal } \\
\left(\alpha_{X}+\mathrm{id}\right) \circ l_{F, X} \circ F \kappa_{2} & =\left(\alpha_{X}+\mathrm{id}\right) \circ \kappa_{2} \circ !_{F 1} & & \text { by (3) } \\
& =\kappa_{2} \circ !_{F 1} & & \\
& =\kappa_{2} \circ !_{G 1} \circ \alpha_{1} & & \\
& =l_{G, X} \circ G \kappa_{2} \circ \alpha_{1} & & \text { by (3) } \\
& =l_{G, X} \circ \alpha_{X+1} \circ F \kappa_{2} & & \text { since } \alpha \text { is }(+, 0) \text {-monoidal } .
\end{aligned}
$$


We define $\alpha_{+1}: F_{+1} \Rightarrow G_{+1}$ by $\left(\alpha_{+1}\right)_{X}=\widehat{\alpha}_{X}: F X \rightarrow G X$. It is natural: for $f: X \rightarrow Y$ in $\mathbf{A}_{+1}$,

$$
\begin{array}{rlrl}
\left(\alpha_{+1}\right)_{Y} \hat{\circ} F_{+1} f & =\widehat{\alpha}_{Y} \hat{\circ}\left(l_{F, Y} \circ F f\right) & \\
& =\left(\alpha_{Y}+\mathrm{id}_{1}\right) \circ l_{F, Y} \circ F f & \\
& =l_{G, Y} \circ \alpha_{X+1} \circ F f & & \text { by (4) } \\
& =l_{G, Y} \circ G f \circ \alpha_{X} & & \text { by naturality of } \alpha \\
& =\left(l_{G, Y} \circ G f\right) \circ \widehat{\alpha}_{X} & \\
& =G_{+1} f \hat{\circ}\left(\alpha_{+1}\right)_{X} . &
\end{array}
$$

It is monoidal with respect to $(+, 0)$ :

$$
\begin{aligned}
\left(\alpha_{+1}\right)_{X+Y} \hat{\circ} F_{+1} \widehat{\kappa}_{1} & =\widehat{\alpha}_{X+Y} \hat{\circ} \widehat{F \kappa_{1}} \\
& =\left(\alpha_{X+Y} \circ F \kappa_{1}\right)^{\Upsilon} \\
& =\left(G \kappa_{1} \circ \alpha_{X}\right)^{\Upsilon} \\
& =\widehat{G \kappa}_{1} \hat{\circ} \widehat{\alpha}_{X} \\
& =G_{+1} \widehat{\kappa}_{1} \hat{\circ}\left(\alpha_{+1}\right)_{X}
\end{aligned}
$$

and similarly we have $\left(\alpha_{+1}\right)_{X+Y} \hat{\circ} F_{+1} \widehat{\kappa}_{2}=G_{+1} \widehat{\kappa}_{2} \hat{\circ}\left(\alpha_{+1}\right)_{Y}$. The arrow $\left(\alpha_{+1}\right)_{1}=\widehat{\alpha}_{1}: F 1 \rightarrow G 1$ is total, hence $1_{G 1} \hat{o}\left(\alpha_{+1}\right)_{1}=1_{F 1}$. Therefore $\alpha_{+1}$ is a 2-cell in FPE.

We then check that $(-)_{+1}: \mathbf{E f f}(\mathbf{A}, \mathbf{B}) \rightarrow \mathbf{F P E}\left(\mathbf{A}_{+1}, \mathbf{B}_{+1}\right)$ is a (1-)functor. For the identity $\operatorname{id}_{F}: F \Rightarrow$ $F$ we have $\left(\left(\operatorname{id}_{F}\right)_{+1}\right)_{X}=\widehat{\mathrm{id}}_{X}: F X \rightarrow F X$, so that $\left(\operatorname{id}_{F}\right)_{+1}=\mathrm{id}_{F_{+1}}: F_{+1} \Rightarrow F_{+1}$. Let $\alpha: F \Rightarrow G$ and $\beta: G \Rightarrow H$ be 2-cells in Eff. Then

$$
\left((\beta \circ \alpha)_{+1}\right)_{X}=\left(\beta_{X} \circ \alpha_{X}\right)^{\uparrow}=\widehat{\beta}_{X} \hat{\circ} \widehat{\alpha}_{X}=\left(\beta_{+1}\right)_{X} \hat{\circ}\left(\alpha_{+1}\right)_{X}=\left(\beta_{+1} \circ \alpha_{+1}\right)_{X} .
$$

Therefore $(\beta \circ \alpha)_{+1}=\beta_{+1} \circ \alpha_{+1}$.

Now we show that $(-)_{+1}$ is a 2 -functor. For the identity functor $\mathrm{id}_{\mathbf{B}}: \mathbf{B} \rightarrow \mathbf{B}$, it is easy to see the canonical isomorphism $l_{\mathrm{id}_{\mathbf{B}}, X}: X+1 \rightarrow X+1$ is the identity, so that $\left(\operatorname{id}_{\mathbf{B}}\right)_{+1}=\operatorname{id}_{\mathbf{B}_{+1}}$. Let $F: \mathbf{A} \rightarrow \mathbf{B}$ and $G: \mathbf{B} \rightarrow \mathbf{C}$ be arrows in Eff. Note the equation

$$
l_{G F, X}=l_{G, F X} \circ G l_{F, X},
$$

which holds because

$$
\begin{gathered}
l_{G, F X} \circ G l_{F, X} \circ G F \kappa_{1}=l_{G, F X} \circ G \kappa_{1}=\kappa_{1}=l_{G F, X} \circ G F \kappa_{1} \\
l_{G, F X} \circ G l_{F, X} \circ G F \kappa_{2}=l_{G, F X} \circ G \kappa_{2} \circ G !_{F 1}=\kappa_{2} \circ !_{G 1} \circ G !_{F 1}=\kappa_{2} \circ !_{G F 1}=l_{G F, X} \circ G F \kappa_{2},
\end{gathered}
$$

using (2) and (3). For $f: X \rightarrow Y$ in $\mathbf{A}_{+1}$, using (5),

$$
(G F)_{+1} f=l_{G F, Y} \circ G F f=l_{G, F Y} \circ G l_{F, Y} \circ G F f=G_{+1}\left(l_{F, Y} \circ F f\right)=G_{+1} F_{+1} f .
$$

Hence $(G F)_{+1}=G_{+1} F_{+1}$. For $\alpha: F \Rightarrow F^{\prime}$, one has

$$
\left((G \alpha)_{+1}\right)_{X}=\widehat{G \alpha_{X}}=G_{+1} \widehat{\alpha}_{X}=\left(G_{+1} \alpha_{+1}\right)_{X},
$$

so that $(G \alpha)_{+1}=G_{+1} \alpha_{+1}$. For $\alpha: G \Rightarrow G^{\prime}$, one has

$$
\left((\beta F)_{+1}\right)_{X}=\widehat{\beta}_{F X}=\left(\beta_{+1}\right)_{F_{+1} X}=\left(\beta_{+1} F_{+1}\right)_{X},
$$

and therefore $(\beta F)_{+1}=\beta_{+1} F_{+1}$. 
Lemma B.3. The mapping $(\mathbf{C}, I) \mapsto \mathbf{C}_{\mathrm{t}}$ for a FinPAC with effects $(\mathbf{C}, I)$ gives rise to a 2-functor $(-)_{\mathrm{t}}: \mathbf{F P E} \rightarrow$ Eff.

Proof. Recall that $\mathbf{C}_{\mathrm{t}}$ is an effectus by Theorem 4.10. Let $F:\left(\mathbf{C}, I_{\mathbf{C}}\right) \rightarrow\left(\mathbf{D}, I_{\mathbf{D}}\right)$ be an arrow in FPE. If $f: X \rightarrow Y$ is a total arrow in $\mathbf{C}$, then

$$
\operatorname{Dp}(F f)=1_{F Y} \circ F f=1_{F I} \circ F 1_{Y} \circ F f=1_{F I} \circ F \operatorname{Dp}(f)=1_{F I} \circ F 1_{X}=1_{F X},
$$

that is, $F f$ is total. Therefore $F$ restricts to the functor $F_{\mathrm{t}}: \mathbf{C}_{\mathrm{t}} \rightarrow \mathbf{D}_{\mathrm{t}}$ in a commutative diagram:

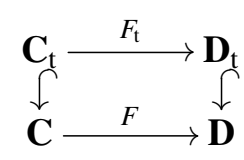

Because $\mathbf{C}_{\mathrm{t}}$ inherits coproducts from $\mathbf{C}$, the functor $F_{\mathrm{t}}$ preserves finite coproducts as $F$ does. Recall that $I_{\mathbf{C}}$ and $I_{\mathbf{D}}$ are the final objects in $\mathbf{C}_{\mathbf{t}}$ and $\mathbf{D}_{\mathrm{t}}$ respectively. By definition we have $F I_{\mathbf{C}} \cong I_{\mathbf{D}}$ in $\mathbf{D}$ and hence in $\mathbf{D}_{\mathrm{t}}$, so that $F_{\mathrm{t}}$ preserves the final object. Therefore $F_{\mathrm{t}}$ is an arrow in Eff.

Let $F, G: \mathbf{C} \rightarrow \mathbf{D}$ be arrows and $\alpha: F \Rightarrow G$ a 2-cell in FPE. Then

$$
\begin{aligned}
\operatorname{Dp}\left(\alpha_{X}\right)=1_{G X} \circ \alpha_{X} & =1_{G I_{\mathrm{C}}} \circ G 1_{X} \circ \alpha_{X} & & \text { since } G \text { is an arrow in FPE } \\
& =1_{G I_{\mathrm{C}}} \circ \alpha_{I_{\mathrm{C}}} \circ F 1_{X} & & \text { by naturality of } \alpha \\
& =1_{F I_{\mathbf{C}}} \circ F 1_{X} & & \text { since } \alpha \text { is a 2-cell in FPE } \\
& =1_{F X} & & \text { since } F \text { is an arrow in FPE },
\end{aligned}
$$

so that $\alpha_{X}$ is total. Hence we can restrict $\alpha$ to the natural transformation $\alpha_{\mathrm{t}}: F_{\mathrm{t}} \Rightarrow G_{\mathrm{t}}$ with $\left(\alpha_{\mathrm{t}}\right)_{X}=\alpha_{X}$, which is obviously a 2-cell in Eff. Then it is easy to see that $(-)_{t}$ gives a (1-)functor $\mathbf{F P E}(\mathbf{C}, \mathbf{D}) \rightarrow$ $\operatorname{Eff}\left(\mathbf{C}_{\mathrm{t}}, \mathbf{D}_{\mathrm{t}}\right)$.

Finally, we can easily check $\left(\operatorname{id}_{\mathbf{C}}\right)_{\mathrm{t}}=\mathrm{id}_{\mathbf{C}_{\mathrm{t}}},(G F)_{\mathrm{t}}=G_{\mathrm{t}} F_{\mathrm{t}},(G \alpha)_{\mathrm{t}}=G_{\mathrm{t}} \alpha_{\mathrm{t}},(\beta F)_{\mathrm{t}}=\beta_{\mathrm{t}} F_{\mathrm{t}}$ for arrows $F: \mathbf{C} \rightarrow \mathbf{D}$ and $G: \mathbf{D} \rightarrow \mathbf{E}$, and 2-cells $\alpha: F \Rightarrow F^{\prime}$ and $\beta: G \Rightarrow G^{\prime}$ in FPE. Therefore $(-)_{\mathrm{t}}$ gives a 2-functor FPE $\rightarrow$ Eff.

Theorem B.4. The 2-functors $(-)_{+1}: \mathbf{E f f} \rightarrow \mathbf{F P E}$ and $(-)_{\mathrm{t}}: \mathbf{F P E} \rightarrow \mathbf{E f f}$ form a 2-equivalence of 2categories $\mathbf{E f f} \simeq$ FPE. Namely, there are 2-natural isomorphisms $\mathrm{id}_{\mathbf{E f f}} \cong\left((-)_{+1}\right)_{\mathrm{t}}$ and $\mathrm{id}_{\mathbf{F P E}} \cong\left((-)_{\mathrm{t}}\right)_{+1}$.

Proof. We write $\Phi_{\mathbf{B}}: \mathbf{B} \rightarrow\left(\mathbf{B}_{+1}\right)_{\mathrm{t}}$ for the isomorphism of categories in Proposition 4.6, which is given by $\Phi_{\mathbf{B}} X=X$ and $\Phi_{\mathbf{B}} f=\widehat{f}$. It preserves finite coproducts and the final object, so that $\Phi_{\mathbf{B}}$ is an arrow in Eff. Let $F: \mathbf{A} \rightarrow \mathbf{B}$ be an arrow in Eff. Because $F_{+1}$ is a lifting of $F$, and $\left(F_{+1}\right)_{\mathrm{t}}$ is a restriction of $F_{+1}$, the following diagram commutes.

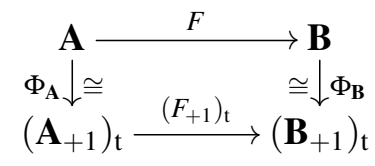

Let $\alpha: F \Rightarrow G$ be a 2-cell in Eff. Then

$$
\left(\left(\alpha_{+1}\right)_{\mathrm{t}} \Phi_{\mathbf{A}}\right)_{X}=\left(\left(\alpha_{+1}\right)_{\mathrm{t}}\right)_{\Phi_{\mathbf{A}} X}=\left(\alpha_{+1}\right)_{X}=\widehat{\alpha}_{X}=\Phi_{\mathbf{B}} \alpha_{X}=\left(\Phi_{\mathbf{B}} \alpha\right)_{X}
$$

so that $\left(\alpha_{+1}\right)_{\mathbf{t}} \Phi_{\mathbf{A}}=\Phi_{\mathbf{B}} \alpha$. Therefore $\Phi$ defines a 2-natural isomorphism id $\operatorname{Eff}_{2} \Rightarrow\left((-)_{+1}\right)_{\mathbf{t}}$.

Next, we write $\Psi_{\mathbf{C}}:\left(\mathbf{C}_{\mathrm{t}}\right)_{+1} \rightarrow \mathbf{C}$ for the isomorphism of categories in Lemma 4.9, which is defined by $\Psi_{\mathbf{C}} X=X$ and $\Psi_{\mathbf{C}} f=\triangleright_{1} \circ f$. It preserves finite coproducts and the unit object $I$, since $I$ is the final 
object of $\mathbf{C}_{\mathrm{t}}$. If we write $1_{X}$ for the top of $\mathbf{C}(X, I)$, then the top of $\left(\mathbf{C}_{\mathrm{t}}\right)_{+1}(X, I)$ is $\widehat{1}_{X}=\kappa_{1} \circ 1_{X}$, and therefore we have $\Psi_{\mathbf{C}} \widehat{1}_{X}=\triangleright_{1} \circ \kappa_{1} \circ 1_{X}=1_{X}$. Hence $\Psi_{\mathbf{C}}$ is an arrow in FPE. Let $F: \mathbf{C} \rightarrow \mathbf{D}$ be an arrow in FPE. Note that the following diagram commutes,

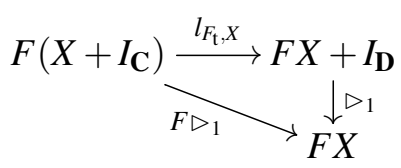

since

$$
\begin{gathered}
\triangleright_{1} \circ l_{F_{\mathrm{t}}, X} \circ F \kappa_{1}=\triangleright_{1} \circ \kappa_{1}=\mathrm{id}_{F X}=F \operatorname{id}_{X}=F \triangleright_{1} \circ F \kappa_{1} \\
\triangleright_{1} \circ l_{F_{\mathrm{t}}, X} \circ F \kappa_{2}=\triangleright_{1} \circ \kappa_{2} \circ 1_{F I_{\mathbf{C}}}=0_{F I_{\mathbf{C}}, F X}=F 0_{I_{\mathbf{C}}, X}=F \triangleright_{1} \circ F \kappa_{2},
\end{gathered}
$$

where $F 0_{I_{\mathbf{C}}, X}=0_{F I_{\mathbf{C}}, F X}$ holds because $F$ preserves the zero object. For $f: X \rightarrow Y$ in $\left(\mathbf{C}_{\mathrm{t}}\right)_{+1}$, we have

$$
\Psi_{\mathbf{D}}\left(F_{\mathrm{t}}\right)_{+1} f=\triangleright_{1} \circ l_{F_{\mathrm{t}}, Y} \circ F f=F \triangleright_{1} \circ F f=F\left(\triangleright_{1} \circ f\right)=F \Psi_{\mathbf{C}} f,
$$

and hence $\Psi_{\mathbf{D}}\left(F_{\mathrm{t}}\right)_{+1}=F \Psi_{\mathbf{C}}$. Let $\alpha: F \Rightarrow G$ be a 2-cell in FPE. Then

$$
\left(\Psi_{\mathbf{D}}\left(\alpha_{\mathrm{t}}\right)_{+1}\right)_{X}=\Psi_{\mathbf{D}}\left(\left(\alpha_{\mathrm{t}}\right)_{+1}\right)_{X}=\triangleright_{1} \circ \widehat{\alpha}_{X}=\alpha_{X}=\alpha_{\Psi_{\mathbf{C} X}}=\left(\alpha \Psi_{\mathbf{C}}\right)_{X}
$$

so that $\Psi_{\mathbf{D}}\left(\alpha_{\mathrm{t}}\right)_{+1}=\alpha \Psi_{\mathbf{C}}$. Therefore $\Psi$ defines a 2-natural isomorphism $\left((-)_{\mathrm{t}}\right)_{+1} \Rightarrow \mathrm{id}_{\mathbf{F P E}}$.

\section{Convex sets over a division effect monoid}

Throughout this section, we let $M$ be a division effect monoid (see Definition 6.3).

Lemma C.1. For $r, s, t, u \in M$ with $r \leq s, s \cdot t \leq u, s \neq 0$ and $u \neq 0$, one has $(r / s) \cdot(s t / u)=r t / u$. In particular $(r / s) \cdot(s / u)=r / u$, by setting $t=1$.

Proof. Since $(r / s) \cdot(s t / u) \cdot u=(r / s) \cdot s \cdot t=r \cdot t$.

Lemma C.2. For each nonzero $t \in M$, the 'multiplication by $t$ ' map $(-) \cdot t: M \rightarrow \downarrow(t)$ is an effect module (over $M$ ) isomorphism, with the inverse $(-) / t: \downarrow(t) \rightarrow M$. In particular, $(-) / t$ is an effect module homomorphism: $0 / t=0 ; t / t=1 ;(r \otimes s) / t=r / t \otimes s / t ;$ and $(r s / t)=r(s / t)$.

Proof. The definition of division says that the map $(-) \cdot t: M \rightarrow \downarrow(t)$ is bijective. It is easy to see that $(-) \cdot t$ is an effect module homomorphism. Therefore, to prove it is an isomorphism, it suffices to show that it reflects the orthogonality: if $r \cdot t \perp s \cdot t$ and $r \cdot t \otimes s \cdot t \leq t$, then $r \perp s$. Since the case $r=0$ is trivial, we assume $r \neq 0$. Then $r \cdot t$ is nonzero too because $(-) \cdot t: M \rightarrow \downarrow(t)$ is bijective. Note that $s^{\perp} \cdot t=t \ominus s \cdot t \geq r \cdot t$ and hence $s^{\perp} \cdot t$ is nonzero as well. Then

$$
r=(r \cdot t) / t=\left((r \cdot t) /\left(s^{\perp} \cdot t\right)\right) \cdot\left(\left(s^{\perp} \cdot t\right) / t\right)=\left((r \cdot t) /\left(s^{\perp} \cdot t\right)\right) \cdot s^{\perp} \leq s^{\perp},
$$

so that $r \perp s$.

The division allows us to construct coproducts in the category $\operatorname{Conv}_{M}$ explicitly, in the same way as the case $M=[0,1]$ done in [7]. First we construct a coproduct of the form $X+1$. For a convex set $X$ over $M$, we define a "lifted" convex set $X_{\bullet}$ as follows.

$$
X_{\bullet}=\{(x, r) \in(X \cup\{\bullet\}) \times M \mid x=\bullet \Longleftrightarrow r=0\} \quad(=X \times(M \backslash\{0\}) \cup\{(\bullet, 0)\})
$$


For a formal convex sum $\sum_{i}\left|\left(x_{i}, r_{i}\right)\right\rangle s_{i} \in \mathcal{D}_{M}\left(X_{\bullet}\right)$, define the actual sum by

$$
\emptyset_{i}\left(x_{i}, r_{i}\right) s_{i}=\left(\emptyset_{i} x_{i}\left(r_{i} s_{i} / t\right), t\right) \quad \text { where: } t=\emptyset_{i} r_{i} s_{i} \text {. }
$$

Note that $\emptyset_{i}\left(r_{i} s_{i} / t\right)=\left(\emptyset_{i} r_{i} s_{i}\right) / t=t / t=1$. The formula (6) is not completely rigorous in the case $t=0$ or $x_{i}=\bullet$, but the meaning will be clear. For example, we often mean $(\bullet, 0)$ by $(e, 0)$ even when $e$ is an expression that does not make sense. Then, the diagram

$$
X \stackrel{\kappa_{1}}{\longrightarrow} X_{\bullet} \stackrel{\kappa_{2}}{\longleftarrow} 1 \quad \text { where: } \kappa_{1}(x)=(x, 1) ; \kappa_{2}(\bullet)=(\bullet, 0)
$$

is a coproduct in $\operatorname{Conv}_{M}$, i.e. $X_{\bullet} \cong X+1$. For $f: X \rightarrow Y$ and $g: 1 \rightarrow Y$, define $[f, g]: X_{\bullet} \rightarrow Y$ by $[f, g](x, r)=f(x) r \otimes g(\bullet) r^{\perp}$.

Let $X$ and $Y$ be convex sets over $M$. Define a (convex) subset $X+Y \subseteq X_{\bullet} \times Y_{\bullet}$ by: $((x, r),(y, s)) \in$ $X+Y \Longleftrightarrow r \perp s$ and $r \oslash s=1$. Then the diagram

$$
X \stackrel{\kappa_{1}}{\longrightarrow} X+Y \stackrel{\kappa_{2}}{\longleftarrow} Y \quad \text { where: } \begin{aligned}
& \kappa_{1}(x)=((x, 1),(\bullet, 0)) \\
& \kappa_{2}(y)=((\bullet, 0),(y, 1))
\end{aligned}
$$

is a coproduct in $\operatorname{Conv}_{M}$. For $f: X \rightarrow Z$ and $g: Y \rightarrow Z$, define $[f, g]: X+Y \rightarrow Z$ by $[f, g]((x, r),(y, s))=$ $f(x) r \otimes g(y) s$.

Finally, we show that $\operatorname{Conv}_{M}$ is an effectus. Note that we use a weaker joint monicity requirement than [7, Definition 12].

Proposition C.3. The category $\mathbf{C o n v}_{M}$ of convex sets over a division effect monoid $M$ is an effectus.

Proof. The category $\operatorname{Conv}_{M}$ has binary coproducts as we described above. It also has the empty convex set $0=\varnothing$ as an initial object (unless $M$ is trivial, i.e. a singleton $\{0\}$; in that case, $\mathbf{C o n v}_{M}$ is a trivial category, which is trivially an effectus), and the singleton convex set 1 as a final object. The pullback requirements are shown in the same way as the case $M=[0,1]$, see [7, Proposition 15].

We now prove that the maps $\left[\kappa_{1}, \kappa_{2}, \kappa_{2}\right],\left[\kappa_{2}, \kappa_{1}, \kappa_{2}\right]: 1+1+1 \rightarrow 1+1$ are jointly monic. It is not hard to see that

$$
1+1 \cong\{(r, s) \in M \times M \mid r \otimes s=1\} \cong M
$$

with coprojections $\kappa_{i}: 1 \rightarrow M$ given by $\kappa_{1}(\bullet)=1$ and $\kappa_{2}(\bullet)=0$. Similarly we have

$$
1+1+1 \cong\{(r, s, t) \in M \times M \times M \mid r \otimes s \otimes t=1\}=: M_{3}
$$

Then the maps $\left[\kappa_{1}, \kappa_{2}, \kappa_{2}\right],\left[\kappa_{2}, \kappa_{1}, \kappa_{2}\right]: M_{3} \rightarrow M$ are given by

$$
\left[\kappa_{1}, \kappa_{2}, \kappa_{2}\right](r, s, t)=\kappa_{1}(\bullet) r \otimes \kappa_{2}(\bullet) s \otimes \kappa_{2}(\bullet) t=r \otimes 0 \otimes 0=r
$$

and similarly $\left[\kappa_{2}, \kappa_{1}, \kappa_{2}\right](r, s, t)=s$. To see they are jointly injective, assume

$$
\left[\kappa_{1}, \kappa_{2}, \kappa_{2}\right](r, s, t)=\left[\kappa_{1}, \kappa_{2}, \kappa_{2}\right]\left(r^{\prime}, s^{\prime}, t^{\prime}\right) \quad \text { and } \quad\left[\kappa_{2}, \kappa_{1}, \kappa_{2}\right](r, s, t)=\left[\kappa_{2}, \kappa_{1}, \kappa_{2}\right]\left(r^{\prime}, s^{\prime}, t^{\prime}\right) \text {. }
$$

Then $r=r^{\prime}, s=s^{\prime}$ and so $t=(r \otimes s)^{\perp}=\left(r^{\prime} \otimes s^{\prime}\right)^{\perp}=t^{\prime}$. Hence $(r, s, t)=\left(r^{\prime}, s^{\prime}, t^{\prime}\right)$. 